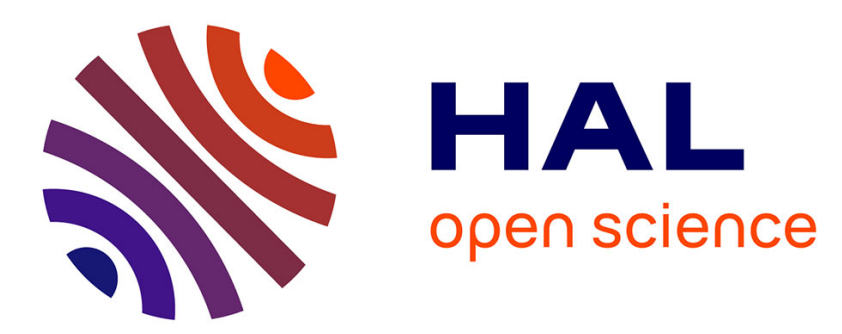

\title{
Maillage de surfaces paramétriques. Partie II: Exemples d'applications
}

Houman Borouchaki, Paul-Louis George

\section{To cite this version:}

Houman Borouchaki, Paul-Louis George. Maillage de surfaces paramétriques. Partie II: Exemples d'applications. [Rapport de recherche] RR-2944, INRIA. 1996. inria-00073755

\section{HAL Id: inria-00073755 https://hal.inria.fr/inria-00073755}

Submitted on 24 May 2006

HAL is a multi-disciplinary open access archive for the deposit and dissemination of scientific research documents, whether they are published or not. The documents may come from teaching and research institutions in France or abroad, or from public or private research centers.
L'archive ouverte pluridisciplinaire HAL, est destinée au dépôt et à la diffusion de documents scientifiques de niveau recherche, publiés ou non, émanant des établissements d'enseignement et de recherche français ou étrangers, des laboratoires publics ou privés. 
$b$

INSTITUT NATIONAL DE RECHERCHE EN INFORMATIQUE ET EN AUTOMATIQUE

\section{Maillage de surfaces paramétriques. Partie II: Exemples d'applications}

Houman Borouchaki - Paul Louis George

$\mathrm{N}^{\circ} 2944$

Juillet 1996

THĖME 4

\section{apport}

de recherche

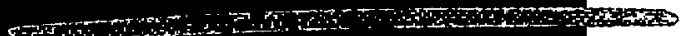


Les rapports de recherche de l'INRIA sont disponibles en format postscript sous ftp.inria.fr (192.93.2.54)

si vous n'ave $z$ pas d'accès ftp la forme papier peut être commandéc par mail : e-mail : dif.gesdif@inria.fr (n'oubliez pas de mentionner votre adresse postale).
INRIA research reports are available in postscript format ftp.inria.fr (192.93.2.54)

if you haven't access by ftp we recommend ordering them by e-mail : e-mail : dif.gesdif@inria.fr (don't forget to mention your postal address).

by mail :

Centre de Diffusion INRIA

BP $105-78153$ Le Chesnay Cedex (FRANCE) BP 105 - 78153 Le Chesnay Cedex (FRANCE) 


\title{
RINRIA
}

\section{Maillage de surfaces paramétriques. Partie II: Exemples d'applications}

\author{
Houman BOROUCHAKI et Paul Louis GEORGE* \\ Thème 4 - Simulation et optimisation \\ de systèmes complexes \\ Projet Gamma
}

Rapport de recherche $n^{\circ} 2944-23$ juillet 1996 - 40 pages

Résumé : Ce papier présente quelques exemples de maillages surfaciques, ainsi que des maillages surfaciques cylindriques, obtenus par une approche de Delaunay anisotrope. Il constitue la partie II de l'étude, la partie I décrivant les aspects théoriques et algorithmiques de la méthode de maillage.

Mots-clé : Triangulation de Delaunay, maillage anisotrope, maillage de surface, maillage adaptatif.

(Abstract: pto)

*E-mail : Paul-Louis.George@inria.fr 


\title{
Parametric Surfaces Meshing. Part II: Application Examples
}

\begin{abstract}
This paper gives some application examples of parametric and cylindrical surface meshes obtained by an anisotropic Delaunay mesh generation method. The paper illustrates part II of a study whose theoretical and algorithmical aspects are describing in a report refered to as part $I$.
\end{abstract}

Key-words: Delaunay triangulation, Anisotropic mesh generation, Surface meshing, Mesh adaption. 


\section{Table des matières}

1 Introduction 4

2 Définition de quelques champs de métriques $\mathbf{5}$

2.1 Maillages uniformes . . . . . . . . . . . . . . 6

2.2 Maillages géométriques . . . . . . . . . . . . . . 6

2.2.1 Métrique isotrope du rayon de courbure minimal . . . . 6

2.2.2 Métrique anisotropes des rayons de courbure principaux 7

2.3 Maillages gouvernés géométriques ... . . . . . . . 7

3 Un exemple analytique $\quad \mathbf{8}$

3.1 Maillages uniformes . . . . . . . . . . . . . . . . . 9

3.2 Maillages géométriques . . . . . . . . . . . . . . . . . . . 12

3.3 Maillages gouvernés . . . . . . . . . . . . . . . 12

3.4 Maillages gouvernés géométriques . . . . . . . . . . . 14

4 Maillage de surfaces cylindriques $\quad 16$

4.1 Maillage d'un cylindre .................. 16

4.1 .1 Une méthode triviale . . . . . . . . . . . . . 18

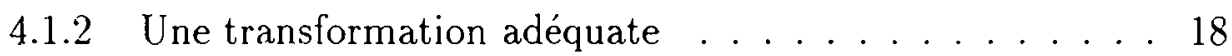

4.2 Un excmple analytique . . . . . . . . . . . . . . 20

4.2.1 Maillages uniformes . . . . . . . . . . . . . 20

4.2 .2 Maillages géométriques ............. . 21

5 Maillage d'un objet numérisé $\mathbf{2 4}$

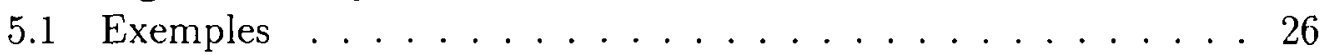

5.1 .1 Maillage du buste de Hugo . . . . . . . . . . . . 26

5.1 .2 Maillage du buste de Voltaire . . . . . . . . . . . . 34

6 Conclusions $\quad 40$

$\mathrm{RR} \mathrm{n}^{\circ} 2944$ 


\section{Introduction}

Nous avons proposé dans [1] une méthode de génération de maillage concernant les surfaces paramétriques. La méthode consiste à générer le maillage dans le domaine des paramètres, et puis à reporter ce dernier sur la surface.

La génération du maillage surfacique peut être gouvernée par un champ continu de métriques défini dans $R^{3}$. Le champ sera défini de telle manière que le maillage souhaité soit un maillage unité (dont toutes les arêtes ont une longueur unité) dans $R^{3}$ muni de la structure riemannienne définie par ce champ. Par suite, si l'on souhaite avoir en un point $P$, des tailles de mesure $h_{1}(P), h_{2}(P)$ et $h_{3}(P)$ suivant les vecteurs définissant une base quelconque $\mathcal{B}(P)$ de $R^{3}$, il suffit de spécifier, en $P$, la métrique

$$
\mathcal{M}_{3}(P)={ }^{t} \mathcal{B}(P)\left(\begin{array}{ccc}
\frac{1}{h_{1}^{2}(P)} & 0 & 0 \\
0 & \frac{1}{h_{2}^{2}(P)} & 0 \\
0 & 0 & \frac{1}{h_{3}^{2}(P)}
\end{array}\right) \mathcal{B}(P)
$$

Par ailleurs, avec cette spécification de métriques, on ne peut que contrôler les tailles suivant trois directions différentes, deux à deux orthogonales. Cette restriction nous a permis de définir une métrique répondant à la spécification, et de proposer un algorithme de construction de maillage basé sur la méthode de Delaunay appliquée dans un contexte riemannien.

Une fois que le champ de métriques est spécifié, on reporte celui-ci dans l'espace des paramètres moyennant une transformation, dépendant des propriétés intrinsèques de la surface, qui conserve la spécification souhaitée dans l'espace des paramètres. Le champ induit est alors défini par les deux premières lignes et les deux premières colonnes de la matrice

$$
{ }^{t} \Pi(P) \mathcal{M}_{3}(P) \Pi(P)
$$

où $\Pi(P)$ est la matrice de passage de la base canonique de $R^{3}$ à la base locale $\mathcal{B}_{l}(P)$ (le plan tangent et la normale) en $P$. Pa construction, ce champ a les propriétés voulues (champ riemannien) et définit à son tour une structure riemannienne dans l'espace des paramètres. Ainsi le problème se ramène à générer 
un maillage unité d'un domaine de $R^{2}$ muni d'une structure riemannienne. Un algorithme, décrit dans [2], est proposé à cette fin.

Le maillage résultant sera conforme au champ spécifié, mais ne donne pas, en général, une approximation satisfaisante de la surface. Par suite, un champ, appelé champ géométrique, est défini qui permet de gouverner la génération d'un maillage qui approche la surface d'une manière satisfaisante. Le champ initial est alors modifié au regard de ce dernier champ, permettant ainsi de gouverner la génération d'un maillage géométrique conforme au champ initial.

En section 2, on rappelle quelques champs naturels de métriques et en particulier le champ géométrique. On montre ensuite comment on peut adapter le champ initialement spécifié pour respecter la géométrie. Section 3, on montre des différents types de maillage d'une surface analytique. Dans la section 4 , on propose une application au cas du maillage des surfaces cylindriques. Section 5 , on montre que l'on peut aussi appliquer les résultats de la section précedente pour mailler un objet "numérisé". Enfin, la dernière section est l'objet d'une rapide conclusion.

\section{Définition de quelques champs de métriques}

Dans cette section on rappelle la définition de quelques champs de métriques servant à gouverner la génération de maillages de différentes natures, par exemple, des maillages

- isotropes de taille constante,

- isotropes gouvernés par le rayon de courbure minimal,

- anisotropes contrôlés par les rayons de courbure principaux,

- gouvernés géométriques.

Le premier champ permet de gouvener la construction d'un maillage uniforme dont les éléments sont tous de taille fixe donnée. Ce champ ne garantit pas, en général, le respect de la géométrie (sauf si la taille est suffisamment petite). Le deuxième et le troisième champ sont propres à la géométrie de la surface et permettent de gouverner la génération d'un maillage correspondant

$\mathrm{RR} \mathrm{n}^{\circ} 2944$ 
à une approximation au second ordre de la surface. Pour le quatrième champ, la construction du maillage est gouvernée à la fois par la géométrie et la spécification voulue. Pour préciser ces différents points, on rappelle l'expression analytique de ces divers champs.

\subsection{Maillages uniformes}

Un maillage uniforme ou $\dot{a} h$ constant d'une surface est un maillage dont les éléments sont tous de taille fixe $h$. Le champ de métriques pour gouverner la génération un tel maillage est de la forme (en chaque point $P$ ),

$$
\mathcal{M}_{3}(P, h)=\left(\begin{array}{ccc}
\frac{1}{h^{2}} & 0 & 0 \\
0 & \frac{1}{h^{2}} & 0 \\
0 & 0 & \frac{1}{h^{2}}
\end{array}\right) \text {. }
$$

\subsection{Maillages géométriques}

Un maillage géométrique d'une surface est un maillage qui approche au mieux la surface. L'approximation considérée ici est du second ordre et le champ de métriques permettant de gouverner la génération d'un tel maillage est défini à partir des rayons de courbure principaux et des directions principales. Deux types de champ sont proposés. Le premier est isotrope et le deuxième est anisotrope.

\subsubsection{Métrique isotrope du rayon de courbure minimal}

Soit $\rho(P)$ le plus petit des rayons de courbure principaux. Le champ de métriques (en chaque point $P$ )

$$
\mathcal{M}_{3}(P, \rho)=\left(\begin{array}{ccc}
\frac{1}{\rho^{2}(P)} & 0 & 0 \\
0 & \frac{1}{\rho^{2}(P)} & 0 \\
0 & 0 & \frac{1}{\rho^{2}(P)}
\end{array}\right)
$$


appelé champ isotrope du rayon de courbure minimale permet de gouverner la génération d'un maillage isotrope géométrique.

\subsubsection{Métrique anisotropes des rayons de courbure principaux}

Soient $\rho_{1}(P)$ et $\rho_{2}(P)$ les deux rayons de courbure principaux et soient $W_{1}(P), W_{2}(P)$ les deux vecteurs unitaires suivant les directions principales correspondantes. Supposons que $\rho_{1}(P) \leq \rho_{2}(P)$ et désignons par

$$
\mathcal{B}_{p}(P)=\left(W_{1}(P), W_{2}(P), W_{1}(P) \times W_{2}(P)\right)
$$

la base locale principale en $P$, où $\times$ désigne le produit vectoriel. Le champ de métriques (en tout point $P$ )

$$
\mathcal{M}_{3}\left(P, \rho_{1}, \rho_{2}\right)={ }^{t} \mathcal{B}_{p}(P)\left(\begin{array}{ccc}
\frac{1}{\rho_{1}^{2}(P)} & 0 & 0 \\
0 & \frac{1}{\rho^{\prime 2}(P)} & 0 \\
0 & 0 & \lambda
\end{array}\right) \mathcal{B}_{p}(P)
$$

appelé champ anisotrope des rayons de courbure principaux, où $\lambda$ est un scalaire quelconque et

$$
\rho_{2}^{\prime}(P)=\rho_{2}(P) \sqrt{1-\left(1-\frac{\rho_{1}(P)}{\rho_{2}(P)}\right)^{2}},
$$

permet de gouverner la génération d'un maillage anisotrope géométrique.

\subsection{Maillages gouvernés géométriques}

Soit un champ quelconque $\mathcal{M}_{3}(P)$. Ce champ n'est pas nécessairement conforme à la définition géométrique de la surface. Pour respecter au mieux ce champ et générer un maillage géométrique on considère alors l'intersection de ce champ avec le champ anisotrope des rayons de courbure principaux $\mathcal{M}_{3}\left(P, \rho_{1}, \rho_{2}\right)$. Celle-ci peut se définir de deux manières différentes

- soit en conservant la forme de la métrique initiale, 
- soit en approchant aux mieux les deux métriques.

La première solution consiste à définir un scalaire $\alpha$ de telle manière que la boule unité correspondant à la métrique intersection

$$
\alpha \mathcal{M}_{3}(P)
$$

soit la plus grande incluse dans la boule unité correspondant à la métrique $\mathcal{M}_{3}\left(P, \rho_{1}, \rho_{2}\right)$.

Quant à la deuxième solution, elle revient à construire la plus grande boule unité incluse à la fois dans la boule unité de $\mathcal{M}_{3}(P)$ et dans celle de $\mathcal{M}_{3}\left(P, \rho_{1}, \rho_{2}\right)$, puis de considérer la métrique correspondante.

\section{Un exemple analytique}

On considère la surface définie par l'équation analytique

$$
(x, y, z=3 \sin (2 x) \cos (2 y))
$$

où le domaine des paramètres est un cercle d'origine $O=(0,0)$ et de rayon 3 dans le plan XY.

Dans un premier temps, le domaine des paramètres est maillé d'une manière isotrope uniforme et les éléments sont presque tous de taille $h=0.2$ (figure 1, en haut à gauche). Ce maillage comprend 876 points et 1746 triangles. En reportant ce maillage sur la surface (figure 1, en haut à droite) on obtient un maillage quelconque de la surface, ce maillage est a priori non conforme avec la géométrie.

Par suite, on va montrer des différents types de maillage de la surface, tous obtenus par une spécification d'un champ de métriques de la forme

$$
\mathcal{M}_{3}(P)=\left(\begin{array}{lll}
a(P) & b(P) & c(P) \\
b(P) & d(P) & e(P) \\
c(P) & e(P) & f(P)
\end{array}\right)
$$

avec $a(P)>0, a(P) d(P)-b^{2}(P)>0$ et $\operatorname{Det}\left(\mathcal{M}_{3}(P)\right)>0$, dans $R^{3}$. Pour chaque type, on donne deux maillages, le premier est le maillage du domaine 
des paramètres et le deuxième, le même maillage reporté sur la surface. Rappelons que le champ $\mathcal{M}_{2}(X)$ induit dans le domaine des paramètres, permettant ainsi de gouverner la génération du maillage, est défini par

$$
\mathcal{M}_{2}(X)=\left({ }^{t} \Pi(P) \mathcal{M}_{3}(P) \Pi(P)\right)_{2 \times 2}
$$

où $\Pi(P)$ est la matrice de passage de la base canonique de $R^{3}$ à la base locale $\mathcal{B}_{l}(P)$ en $P$. Pour $P=(x, y, 3 \sin (2 x) \cos (2 y))$, on a

$$
\Pi(P)=\left(\begin{array}{ccc}
1 & 0 & -\frac{\alpha(P)}{\sqrt{\alpha^{2}(P)+\beta^{2}(P)+1}} \\
0 & 1 & -\frac{\beta(P)}{\sqrt{\alpha^{2}(P)+\beta^{2}(P)+1}} \\
\alpha(P) & \beta(P) & 1
\end{array}\right)
$$

où $\alpha(P)=6 \cos (2 x) \cos (2 y)$ et $\beta(P)=-6 \sin (2 x) \sin (2 y)$. On en déduit que

$$
\mathcal{M}_{2}(X)=\left(\begin{array}{ll}
a^{\prime}(P) & b^{\prime}(P) \\
b^{\prime}(P) & c^{\prime}(P)
\end{array}\right)
$$

avec

$$
\begin{gathered}
a^{\prime}(P)=a(P)+2 c(P) \alpha(P)+f(P) \alpha^{2}(P), \\
b^{\prime}(P)=b(P)+e(P) \alpha(P)+c(P) \beta(P)+f(P) \alpha(P) \beta(P), \\
c^{\prime}(P)=d(P)+2 e(P) \beta(P)+f(P) \beta^{2}(P) .
\end{gathered}
$$

\subsection{Maillages uniformes}

On spécifie les champs isotropes uniformes $\mathcal{M}_{3}(P, 0.4), \mathcal{M}_{3}(P, 0.2)$ et enfin $\mathcal{M}_{3}(P, 0.1)$ et on obtient respectivement les maillages des figures 1 (en bas) ct 2 . Les caractéristiques de ces maillages sont résumées dans le tableau 1 où $n p$ est le nombre de points, $n t$ le nombre de triangles, $t_{C P U}$ le temps CPU (HP735/99Mhz) nécessaire à la génération du maillage et $e$ est l'étirement maximal spécifié (qui correspond au rapport de la plus grande taille spécifiée sur la plus petite suivant toutes les directions) dans le domaine des paramètres.

Ces maillages ne sont pas conformes à la géométrie, mais respectent la spécification de métriques donnée comme on le voit sur les figures.

$\mathrm{RR} \mathrm{n}^{\circ} 2944$ 
H. Borouchaki et P. L. George

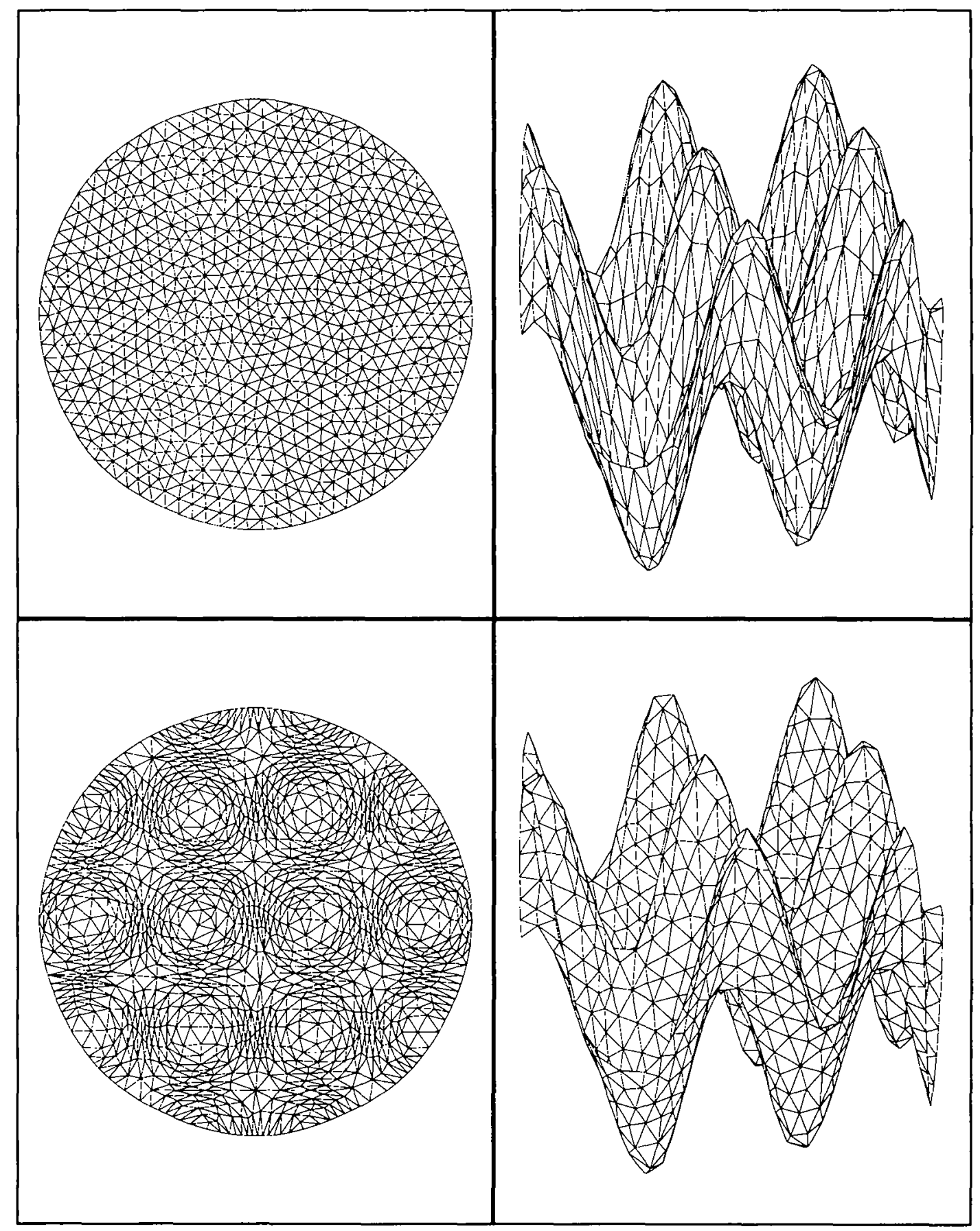

FIG. 1 - Maillage initial et maillage uniforme $(h=0.4)$.

INRIA 


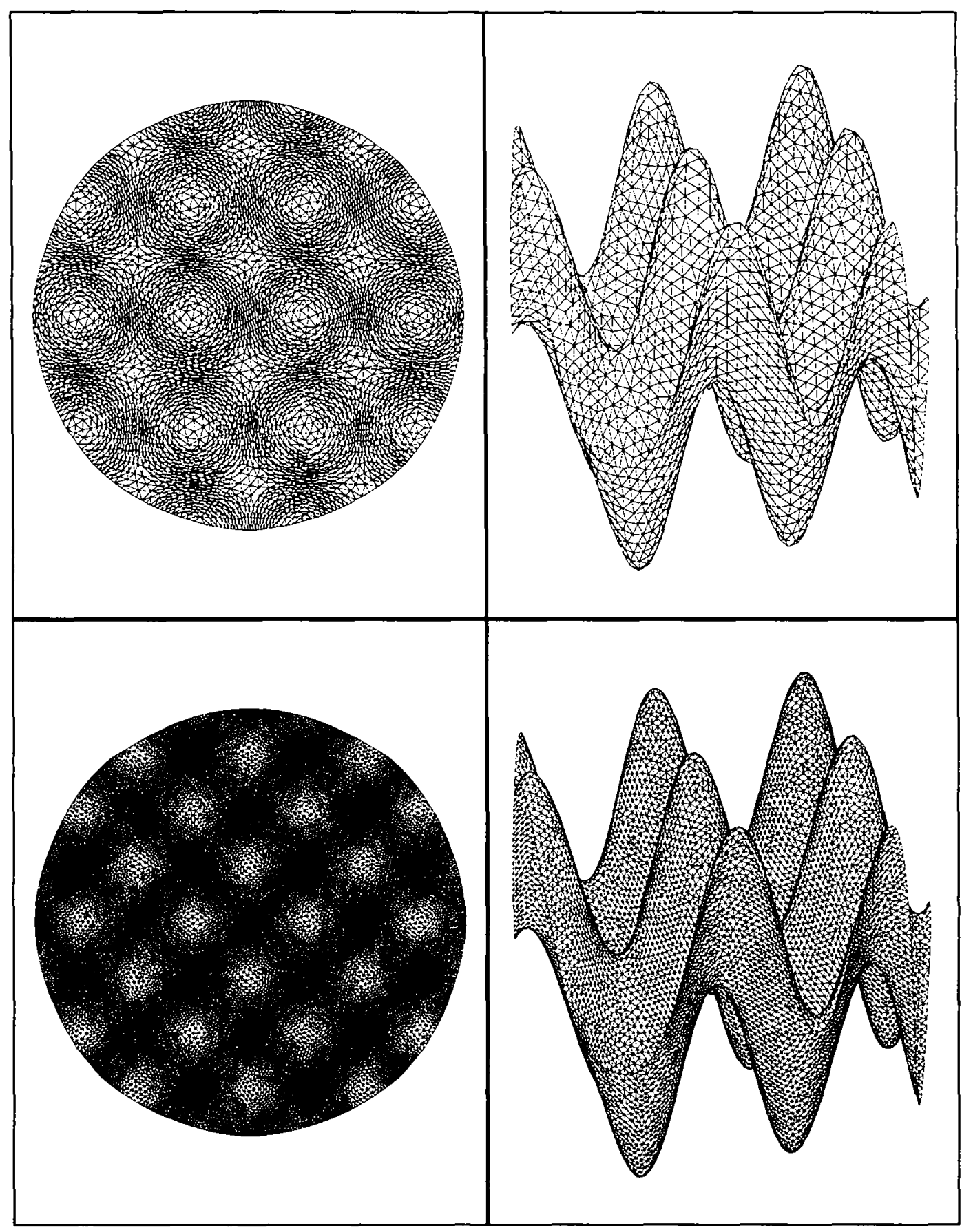

FIG. 2- Maillages uniformes $(h=0.2$ et $h=0.1)$. $\mathrm{RR} \mathrm{n}^{\circ} 2944$ 


\begin{tabular}{|l|c|c|c|c|}
\hline Champ & $n p$ & $n t$ & $t_{C P U}$ & $\mathrm{e}$ \\
\hline $\mathcal{M}_{3}(P, 0.4)$ & 1098 & 2190 & 1.4 & 6. \\
\hline $\mathcal{M}_{3}(P, 0.2)$ & 3905 & 7804 & 4. & 6. \\
\hline $\mathcal{M}_{3}(P, 0.1)$ & 14839 & 29672 & 12. & 6. \\
\hline
\end{tabular}

TAB. 1 - Statistiques relatives aux champs uniformes.

\subsection{Maillages géométriques}

On considère les deux champs de métriques géométriques isotrope $\mathcal{M}_{3}(P, \rho)$ et anisotrope $\mathcal{M}_{3}\left(P, \rho_{1}, \rho_{2}\right)$, tout deux évalués avec la contrainte

$$
\rho_{\min }(=0.05) \leq \rho(P) \leq \rho_{\max }(=2.0)
$$

pour éviter une dégénérescence éventuelle de la taille des éléments. Les maillages résultants sont montrés sur les figures 3 et les statistiques correspondantes sont. reportées dans le tableau 2. Par définition, ces maillages sont conformes à la géométrie et représentent une approximation à l'ordre deux de la surface.

\begin{tabular}{|l|r|r|r|r|}
\hline Champ & $n p$ & $n t$ & $t_{C P U}$ & $\mathrm{e}$ \\
\hline $\mathcal{M}_{3}(P, \rho)$ & 15464 & 30922 & 12. & 6. \\
\hline $\mathcal{M}_{3}\left(P, \rho_{1}, \rho_{2}\right)$ & 2243 & 4480 & 2. & 8. \\
\hline
\end{tabular}

TAB. 2 - Statistiques relatives aux champs géométriques.

\subsection{Maillages gouvernés}

On considère deux champs de métriques dont le premier $\mathcal{M}_{3}(P$, iso $)$ est isotrope et est défini par

$$
\mathcal{M}_{3}(P, \text { iso })=\left(\begin{array}{ccc}
\frac{1}{f^{2}(r)} & 0 & 0 \\
0 & \frac{1}{f^{2}(r)} & 0 \\
0 & 0 & \frac{1}{f^{2}(r)}
\end{array}\right)
$$




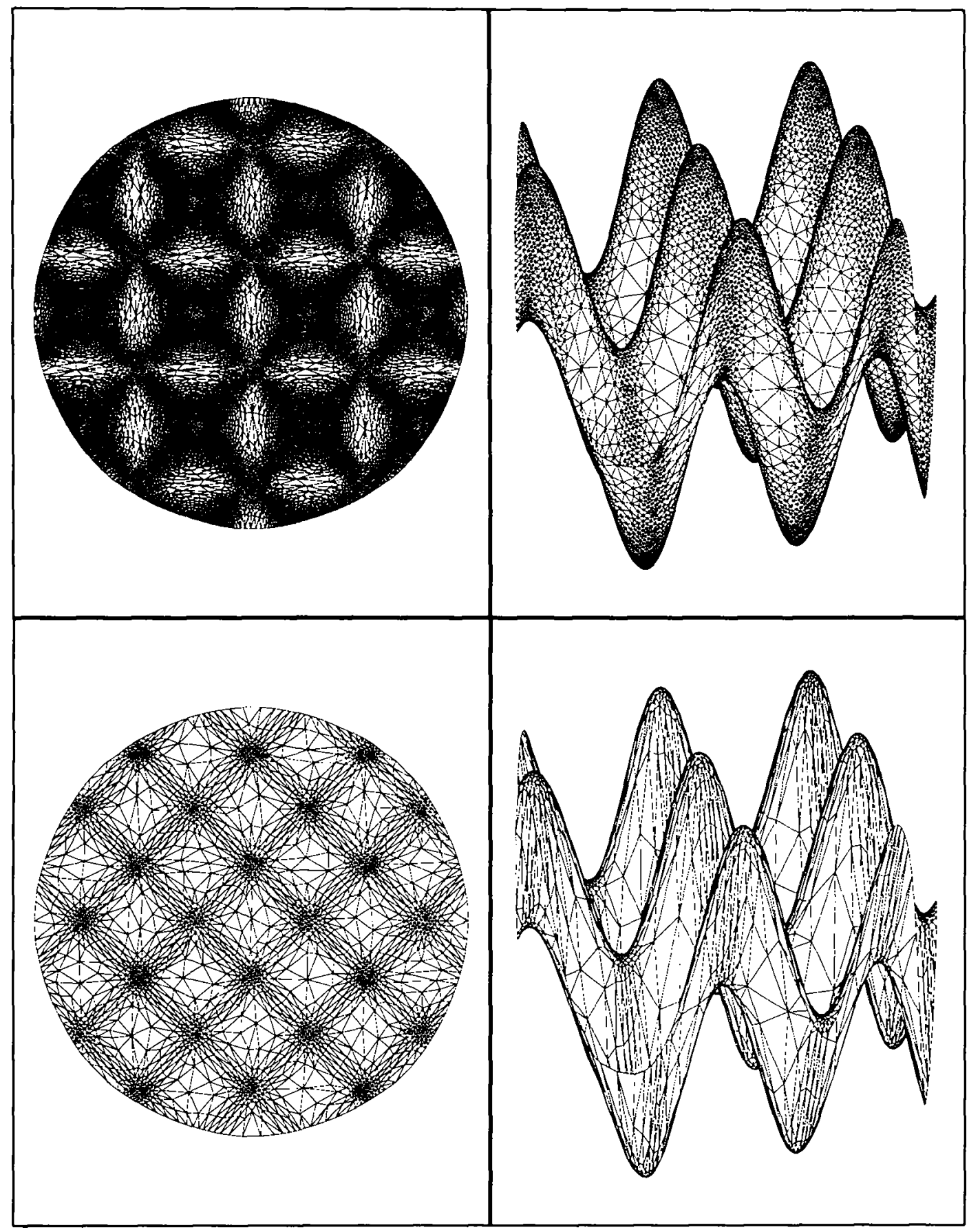

FIG. 3 - Máillages géométriques isotropes et anisotropes.

$\mathrm{RR} \mathrm{n}^{\circ} 2944$ 
où $f(r)=0.05\left(1+r^{2}\right)$ avec $r^{2}=x^{2}+y^{2}$, et le deuxième $\mathcal{M}_{3}(P$, aniso $)$, anisotrope, est défini par

$$
\mathcal{M}_{3}(P, \text { aniso })=\left(\begin{array}{ccc}
\frac{1}{0.02^{2}} & 0 & 0 \\
0 & \frac{1}{0.02^{2}} & 0 \\
0 & 0 & \frac{1}{g^{2}(z)}
\end{array}\right)
$$

où $g(z)=0.1\left|z^{2}-2.25\right|+0.01$.

Le premier champ indique une croissance quadratique en taille par rapport à $x$ et $y$, il correspond à la demande d'une taille de plus en plus grande quand on s'éloigne de l'origine. Par ailleurs, une taille minimale de 0.05 est imposée. Quant au deuxième champ, il traduit la demande d'éléments de plus en plus étirés lorsque l'on s'approche des deux plans $z=1.5$ et $z=-0.5$, avec un étirement maximal de 20. Les figures 4 en haut (resp. 4 en bas) montrent les maillages correspondants au champ $\mathcal{M}_{3}\left(P\right.$, iso) (resp. $\mathcal{M}_{3}(P$, aniso)) et le tableau 3 donne les statistiques correspondantes. Dans les deux cas, les maillages résultants ne sont pas conformes à la géométrie mais respectent les champs spécifiés.

\begin{tabular}{|l|r|r|r|r|}
\hline Champ & $n p$ & $n t$ & $t_{C P U}$ & $\mathrm{e}$ \\
\hline $\mathcal{M}_{3}(P$, iso $)$ & 6065 & 12124 & 6. & 6. \\
\hline $\mathcal{M}_{3}(P$, aniso $)$ & 9279 & 18552 & 17. & 95. \\
\hline
\end{tabular}

TAB. 3 - Statistiques relatives aux champs donnés.

\subsection{Maillages gouvernés géométriques}

On reconsidère les deux champs $\mathcal{M}_{3}(P$, iso $), \mathcal{M}_{3}(P$, aniso $)$ qui vont être modifiés respectivement au regard des champs $\mathcal{M}_{3}(P, \rho)$ et $\mathcal{M}_{3}\left(P, \rho_{1}, \rho_{2}\right)$ pour mieux respecter la géométrie. Ainsi le premier, $\mathcal{M}_{3}(P$, iso,géom), est déduit de $\mathcal{M}_{3}(P$, iso $)$ en remplaçant $f(r)$ par $\min (f(r), \rho(P))$ où $P=(x, y, z)$ avec $z=3 \sin (2 x) \cos (2 y))$ et $\rho(P)$ est le rayon de courbure minimal en $P$. Quant au deuxième, $\mathcal{M}_{3}(P$, aniso,géom), il est obtenu par un procédé d'intersection du 


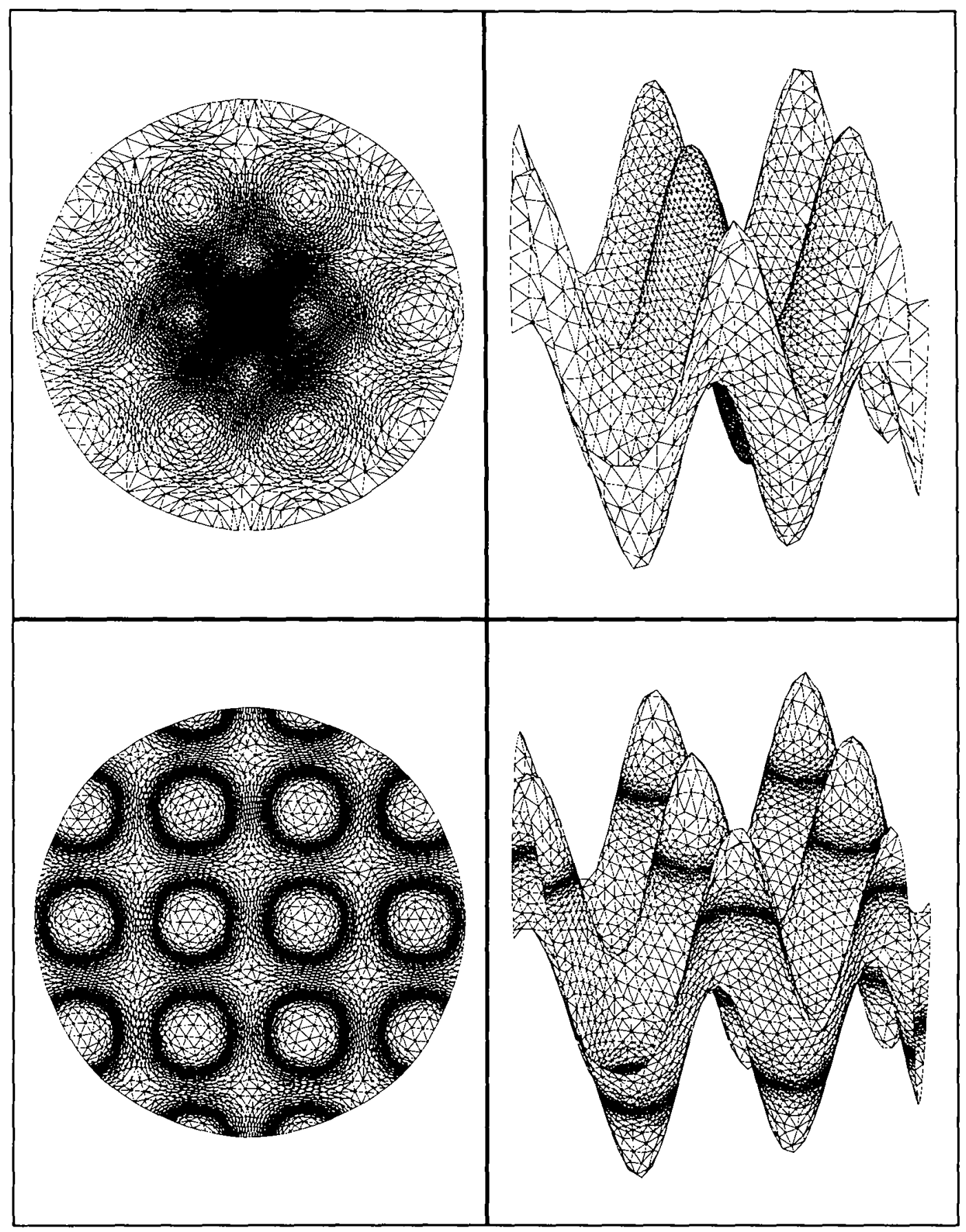

FIG. 4 - Maillages gouvernés.

$\mathrm{RR} \mathrm{n}^{\circ} 2944$ 
champ initial $\mathcal{M}_{3}(P$, aniso $)$ avec le champ anisotrope des rayons de courbure $\mathcal{M}_{3}\left(P, \rho_{1}, \rho_{2}\right)$.

Les figures 5 montrent les maillages résultants. Les statistiques sur ces maillages sont données dans le tableau 4. On constate que la spécification n'est pas respectée par endroit à cause des contraintes géométriques.

\begin{tabular}{|l|r|r|r|r|}
\hline Champ & $n p$ & $n t$ & $t_{C P U}$ & $\mathrm{e}$ \\
\hline $\mathcal{M}_{3}$ (iso,géom) & 17192 & 34378 & 14. & 6. \\
\hline $\mathcal{M}_{3}$ (aniso,géom) & 32212 & 64418 & 45. & 97. \\
\hline
\end{tabular}

TAB. 4 - Statistiques relatives aux champs gouvernés géométriques.

\section{Maillage de surfaces cylindriques}

Une surface est appelée cylindrique s'il existe un difféomorphisme de classe $C^{2}$ entre la surface est un cylindre. Le problème du maillage d'une telle surface se ramène au problème du maillage d'un cylindre grâce au difféomorphisme mettant en relation ces deux surfaces. Par suite, on propose deux méthodes pour le maillage d'un cylindre et on donne un exemple simple de maillages d'une surface cylindrique.

\subsection{Maillage d'un cylindre}

Un cylindre d'axe $\mathrm{OZ}$ et de "rayon" $r$ peut s'écrire sous la forme paramétrique

$$
\begin{gathered}
x=f_{1}(\theta, z)=r \cos (\theta), \\
y=f_{2}(\theta, z)=r \sin (\theta), \\
z=f_{3}(\theta, z)=z
\end{gathered}
$$

où $\theta \in R$ et $z(0 \leq z \leq d)$ constituent les paramètres. Le maillage de ce cylindre via cet espace de paramètres pose un problème dans la mesure où cet espace est périodique et ne constitue pas un domaine de $R^{2}$. En effet les points de coordonnées $(\theta, z)$ et $(\theta+2 \pi, z)$ sont identiques. Pour se faire, on propose deux méthodes dont 


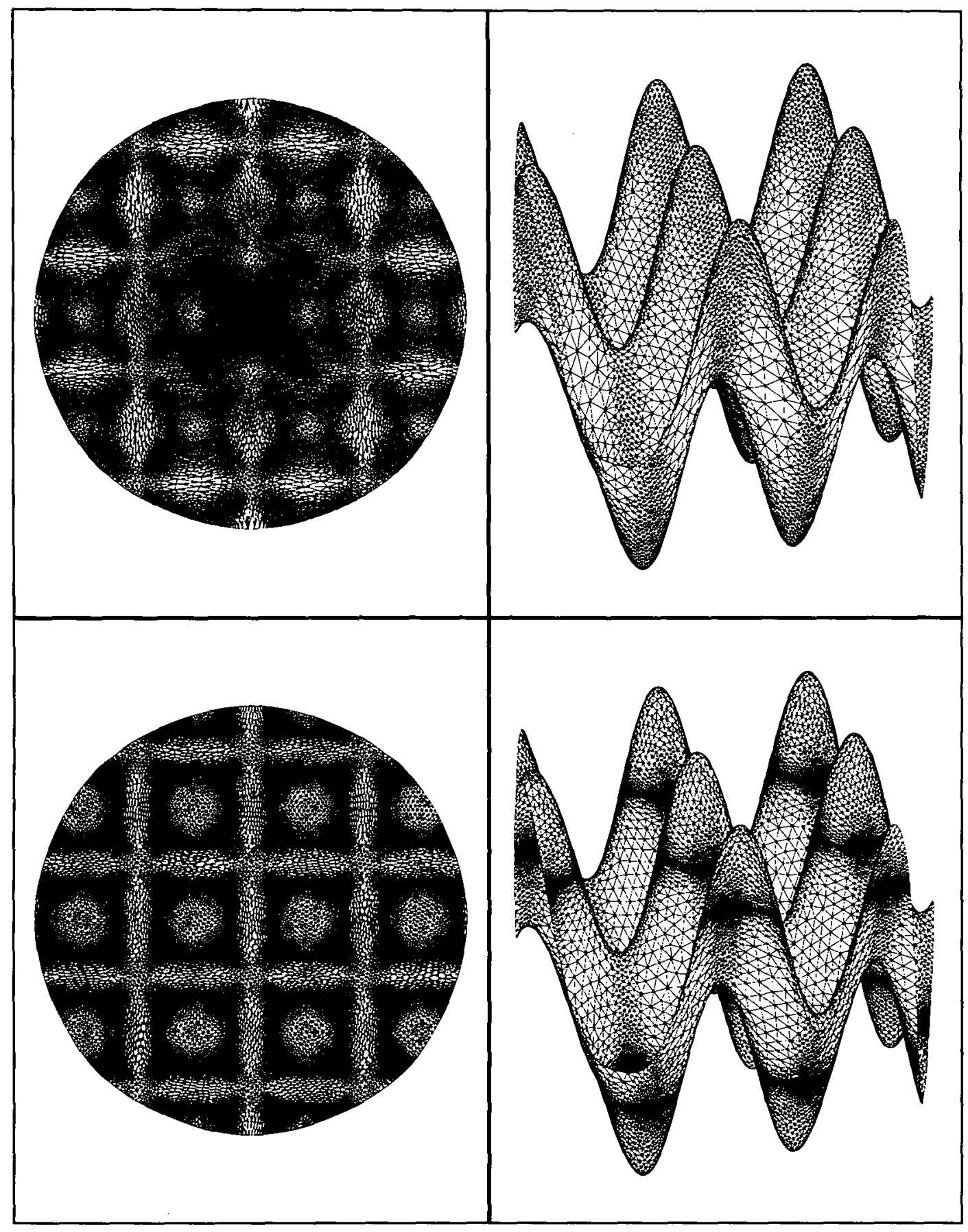

FIG. 5 - Maillages gouvernés géométriques.

$\mathrm{RR} \mathrm{n}^{\circ} 2944$ 
- la première consiste à forcer une contrainte, traduisant la périodicité et

- la deuxième, à changer l'espace des paramètres pour prendre en compte automatiquement la périodicité,

qui vont être détaillées par la suite.

\subsubsection{Une méthode triviale}

La méthode consiste à restreindre l'espace des paramètres au rectangle défini par

$$
\begin{gathered}
0 \leq \theta \leq 2 \pi \\
0 \leq z \leq d
\end{gathered}
$$

ct à s'assurer que la discrétisation du contour de ce rectangle soit identique sur les côtés $\theta=0$ et $\theta=2 \pi$. Ceci revient à imposer une ligne contrainte tracée sur l'objet. Le domaine des paramètres étant bien défini, on peut appliquer la méthode de maillage dans l'espace des paramètres avant de le reporter sur la surface.

\subsubsection{Une transformation adéquate}

Une inćthode plus élégante est de redéfinir l'espace des paramètres pour "casser" la périodicité présente dans la définition des paramètres. En effet, on peut définir le nouvel espace par projection stéréographique d'un point $Q=(0,0, L)$, où $L>d$ sur le plan $z=0$ (figure 6 ). Les nouveaux paramètres sont alors définis par

$$
\begin{aligned}
& x^{\prime}=\frac{L r}{L-z} \cos (\theta) \\
& y^{\prime}=\frac{L r}{L-z} \sin (\theta) .
\end{aligned}
$$

Le nouveau domaine des paramètres est une couronne définie par les deux cercles de centres $O$ et de rayons respectivement $r$ et $\frac{L r}{L-d}$ et le cylindre est 


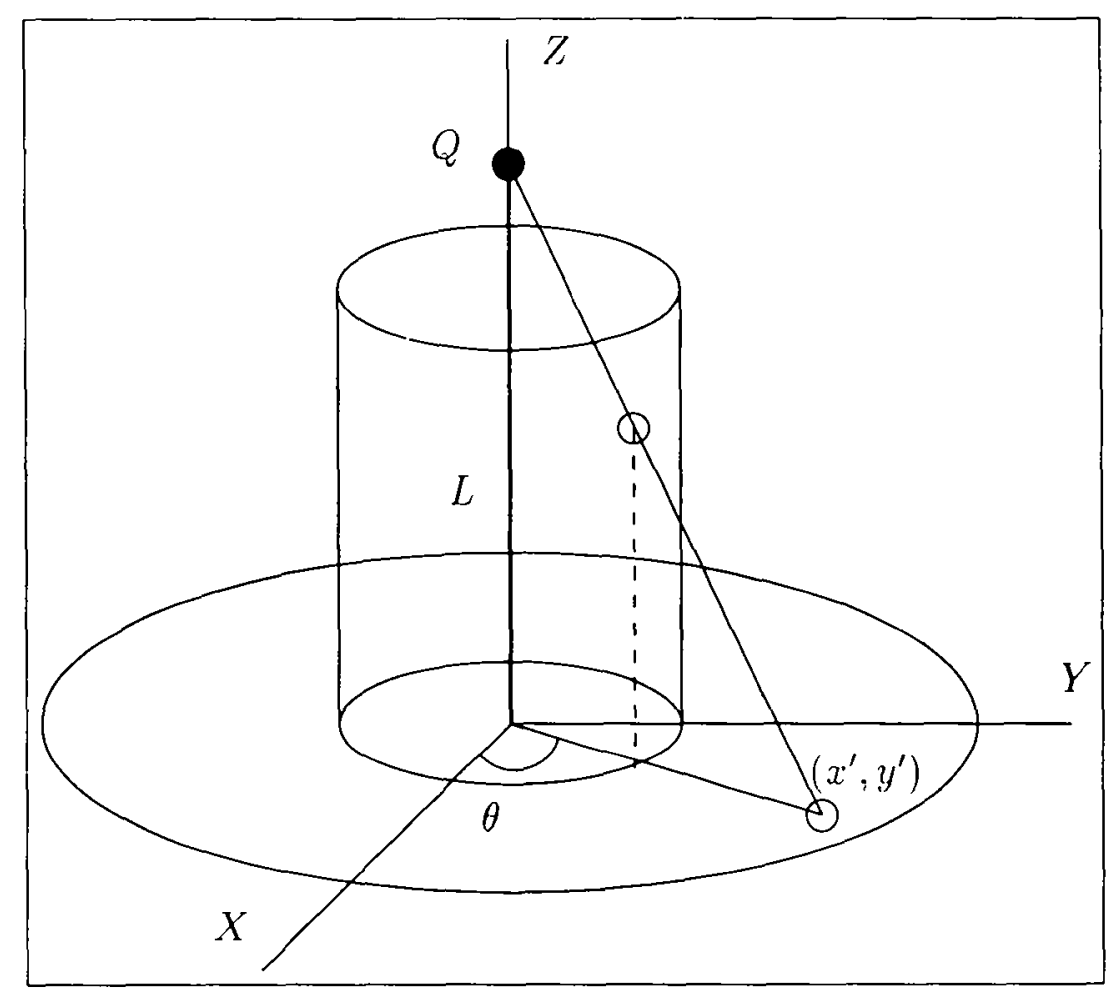

FIG. 6 - Projection sur le plan $z=0$. 
défini via ce domaine par

$$
\begin{gathered}
x=r \frac{x^{\prime}}{\sqrt{x^{\prime 2}+y^{\prime 2}}} \\
y=r \frac{y^{\prime}}{\sqrt{x^{\prime 2}+y^{\prime 2}}} \\
z=L\left(1-\frac{r}{\sqrt{x^{\prime 2}+y^{\prime 2}}}\right) .
\end{gathered}
$$

\subsection{Un exemple analytique}

Dans cette section, on montre des différents types de maillage d'une surface cylindrique (un vase) obtenue par rotation d'un segment courbe de $R^{3}$ autour de l'axe des $Z$. Pour simplifier, le segment courbe est défini de telle manière que sa projection orthogonale sur l'axe des $Z$ soit bijective. Ainsi cette surface est décrite par les équations

$$
\begin{gathered}
x=\delta(z) \cos (\theta), \\
y=\delta(z) \sin (\theta), \\
z=z,
\end{gathered}
$$

où $\delta(z)$ est la fonction définissant le segment courbe tandis que $\theta \in R$ et $z$ $(0 \leq z \leq d)$ constituent les paramètres.

En projetant la surface par rapport à l'axe des $Z$ sur un cylindre de rayon $r$ quelconque et d'axe $O Z$, le problème du maillage de la surface se ramène au problème du maillage du cylindre. Par la suite on va montrer des différents types de maillage de cette surface obtenus en utilisant les deux méthodes décrites ci-dessus. On applique la première méthode pour la construction des maillages uniformes et la deuxième, pour la construction des maillages géométriques.

\subsubsection{Maillages uniformes}

D'après la première méthode il suffit de considérer pour le domaine des paramètres, le rectangle

$$
\begin{gathered}
0 \leq \theta \leq 2 \pi \\
0 \leq z \leq d
\end{gathered}
$$


et de reporter la spécification de métriques correspondant au côté $\theta=0$, sur le côté $\theta=2 \pi$ du rectangle.

En discrétisant ce rectangle, à pas contant, respectivement suivant l'axe des $\theta$ et l'axe des $z$, on obtient un maillage régulier (figure 7 à gauche) dans le domaine des paramètres, dont le report sur la surface (figure 8 en haut à gauche) est un maillage a priori quelconque. Le reste des figures 7 montrent des maillages gouvernés du domaine des paramètres dont les reports sur la surface (le reste des figures 8) sont des maillages uniformes avec des différentes tailles spécifiées. Les statistiques correspondantes à ces maillages sont reportées dans le tableau 5 .

\begin{tabular}{|l|c|c|c|c|}
\hline Champ & $n p$ & $n t$ & $t_{C P U}$ & $\mathrm{e}$ \\
\hline uniforme $h$ & 1214 & 2325 & 1. & 20. \\
\hline uniforme $\frac{h}{2}$ & 4736 & 9265 & 4. & 20. \\
\hline uniforme $\frac{h}{4}$ & 18703 & 36996 & 13. & 20. \\
\hline
\end{tabular}

TAB. 5 - Statistiques relatives aux maillages uniformes du vase.

\subsubsection{Maillages géométriques}

D'après la deuxième méthode, il suffit de coupler les équations (22) avec les équations

$$
\begin{gathered}
\theta=\arctan \left(\frac{y^{\prime}}{x^{\prime}}\right), \\
z=L\left(1-\frac{r}{\sqrt{x^{\prime 2}+y^{\prime 2}}}\right),
\end{gathered}
$$

et de mailler le domaine des paramètres $\left(x^{\prime}, y^{\prime}\right)$ décrivant une couronne définie par deux cercles de centre $O$ et de rayons respectivement $r$ et $\frac{L r}{L-d}$ (la transformation (24) est la projection par rapport au point $(0,0, L)$ sur le plan $z=0$ du cylindre).

Les figures 9 montrent les maillages de la couronne, ainsi que leurs reports sur la surface, respectivement dans les cas géométriques isotropes et géométriques anisotropes. Les statistiques correspondantes sont rcportécs dans le tableau 6.

$\mathrm{RR} \mathrm{n}^{\circ} 2944$ 


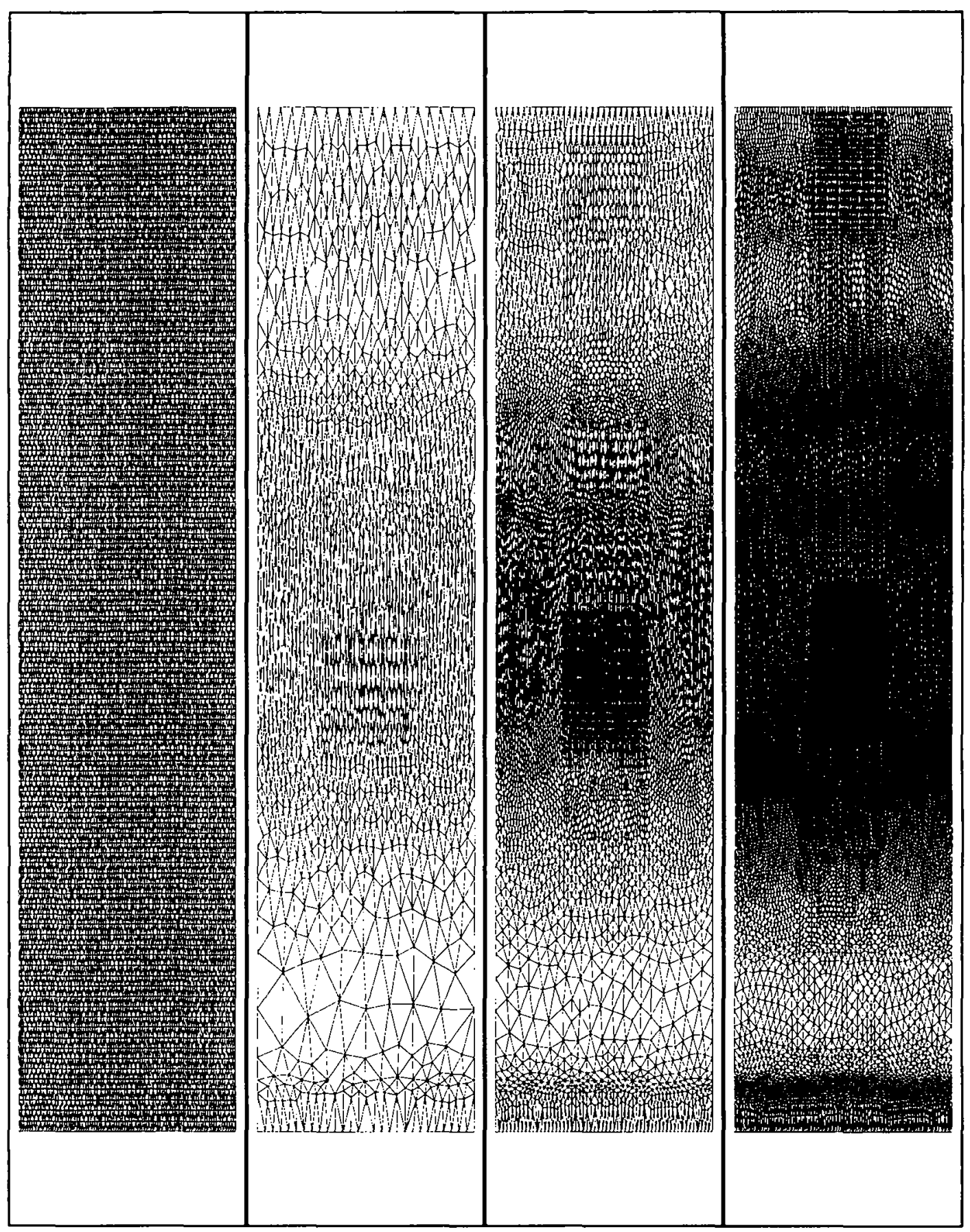

FIG. 7 - Maillage régulier et maillages gouvernés du rectangle. 


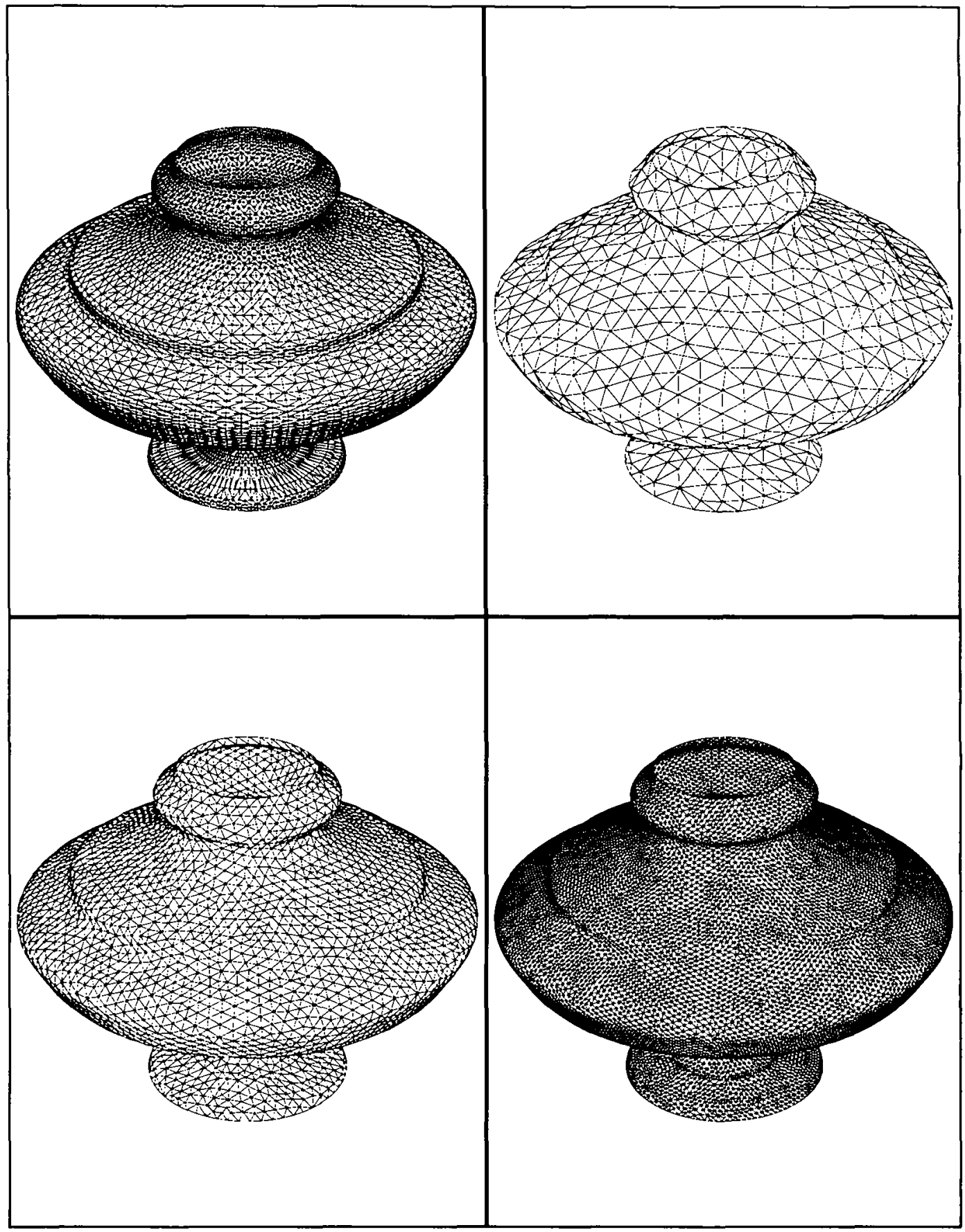

FIG. 8 - Maillage quelconque et maillages uniformes du vase.

$\mathrm{RR} \mathrm{n}^{\circ} 2944$ 


\begin{tabular}{|l|c|c|c|c|}
\hline Champ & $n p$ & $n t$ & $t_{C P U}$ & $\mathrm{e}$ \\
\hline géométrique isotrope & 19133 & 38050 & 16. & 33. \\
\hline géométrique anisotrope & 2300 & 4386 & 3. & 150. \\
\hline
\end{tabular}

TAB. 6 - Statistiques relatives aux maillages géométriques du vase.

On constate qu'en utilisant la deuxième méthode, les étirements maximaux imposés sont plus importants que ceux imposés par rapport à la première méthode. Ceci est dû au fait que la projection stéréographique transforme les métriques initiales en des métriques beaucoup plus allongées.

Remarquons que le nombre d'éléments pour les maillages géométriques dépend des valeurs de tailles minimales et maximales imposées et qu'en modifiant celles-ci on peut éventucllement générer un maillage ayant un nombre d'éléments moins important mais ceci a pour conséquence la dégradation de la qualité des éléments.

\section{Maillage d'un objet numérisé}

On s'intéresse au problème de maillage de la surface d'un objet réel, à partir d'un ensemble de points échantillonnés, obtenues par le système de numérisation tridimensionnelle "3D Videolaser" ([3]). Par son principe de fonctionnement, les données possèdent une topologje rectangulaire, c'est-à-dire, que les points échantillonnés sont répartis sur un réseau de méridiens et de parallèles de la surface de l'objet. En particulier, pour les objets ayant une géométrie cylindrique, les données (points échantillonnés sur la surface) ont une structure matricielle dont les colonnes et les lignes correspondent respectivement aux méridiens et aux parallèles et la valeur associée à un élément (point) de cette matrice représente la distance de ce point à l'axe de rotation de l'objet.

Ainsi ce système permet de définir une surface cylindrique, "d'une manière discrète", par les équations

$$
\begin{gathered}
x=r(\theta, z) \cos (\theta), \\
y=r(\theta, z) \sin (\theta), \\
z=z
\end{gathered}
$$




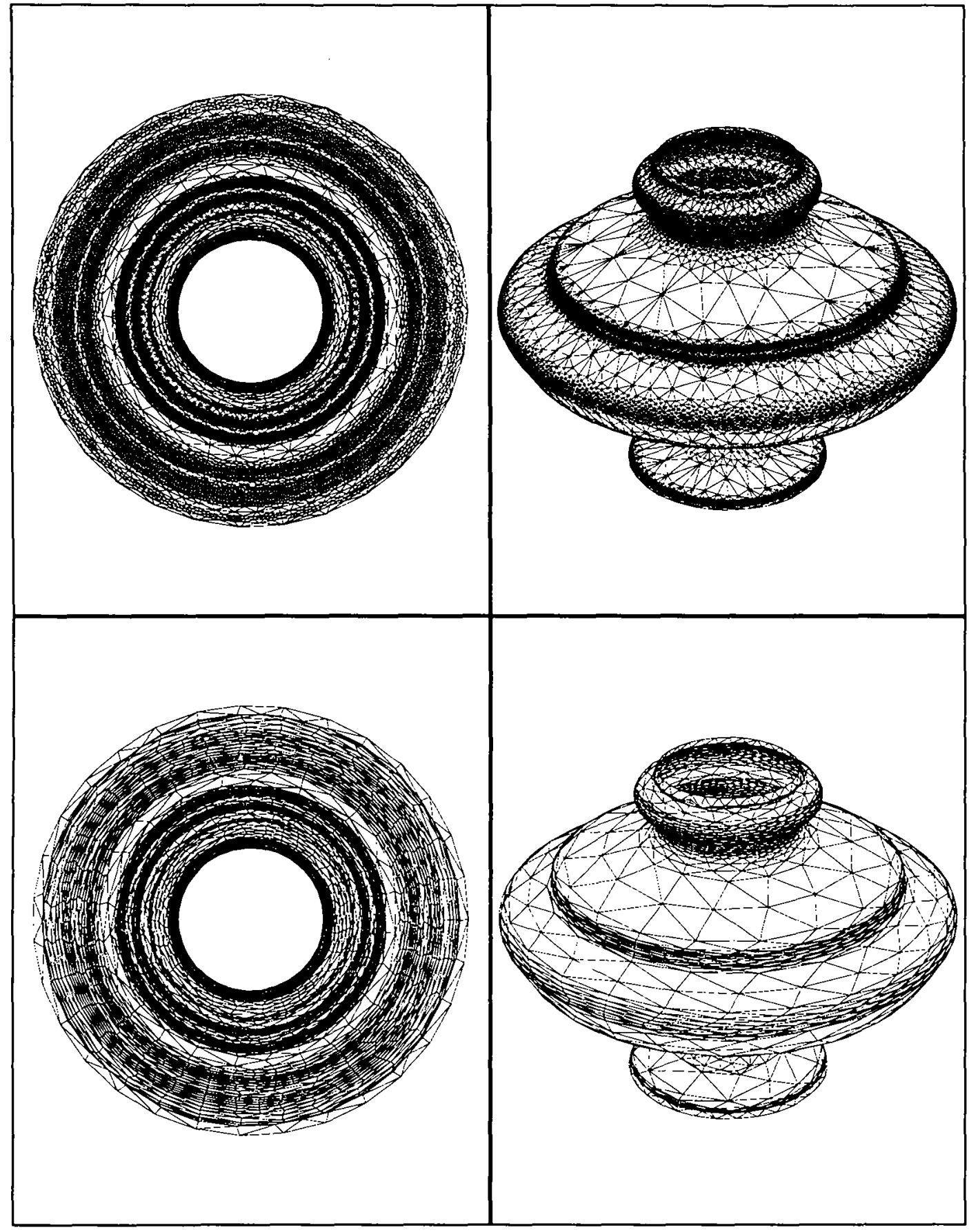

FIG. 9 - Maillages géométriques du vase.

$\mathrm{RR} \mathrm{n}^{\circ} 2944$ 
où le domaine des paramètres est le rectangle

$$
0 \leq \theta<2 \pi \quad \text { et } \quad 0 \leq z \leq d
$$

En effet, la fonction $r(\theta, z)$ est connue uniquement en un nombre fini de points du rectangle, plus précisément sur la grille régulière

$$
\begin{array}{ll}
\theta_{j}=j \Delta \theta & 0 \leq i \leq n c \\
z_{i}=i \Delta z & 0 \leq j \leq n l
\end{array}
$$

où $n c$ (resp. $n l$ ) est le nombre de colonnes (resp. lignes) de la grille et $\Delta \theta$ (resp. $\Delta z$ ) est le pas d'échantillonnage suivant les $\theta$ (resp. $z$ ).

Le maillage d'une telle surface par l'une ou l'autre méthode demande de connaître en chaque sommet de la grille les quantités $r_{\theta}^{\prime}(\theta, z), r_{z}^{\prime}(\theta, z), r_{\theta^{2}}^{\prime \prime}(\theta, z)$, $r_{z^{2}}^{\prime \prime}(\theta, z)$ et $r_{\theta z}^{\prime \prime}(\theta, z)$. Ces dernières sont évaluées d'une manière triviale par une méthode de différences finies.

\subsection{Exemples}

On considère deux exemples de surfaces échantillonnées par le système décrit plus haut. La première, le buste de Hugo, comprend $(120 \times 69)$ échantillons, quant à la deuxième, le buste de Voltaire, $(180 \times 144)$ échantillons. Par la suite, on montre des différents types de maillage de ces objets en utilisant les techniques de maillage de surfaces cylindriques décrites plus haut.

\subsubsection{Maillage du buste de Hugo}

On utilise la première méthode. La figure 10 montre un maillage régulier de la grille Hugo ainsi que son report sur la surface, servant d'espace de contrôle pour les autres maillages gouvernés de l'objet. Les figures 11, 12, 13 et 14 montrent des maillages uniformes avec des pas proportionnels. Les figure 15 et 16 montrent des maillages géométriques. Les étirements imposés, dans le domaine des paramètres, ne sont pas cohérents avec les maillages illustrés. En effet, pour que ces maillages soient plus visibles, un facteur d'echelle est introduit suivant l'axe horizontal (en fait cet axe représente les $\theta$ en degré, et non pas en radian). Les statistiques relatives à ces maillages sont reportées dans le tableau 7 . 


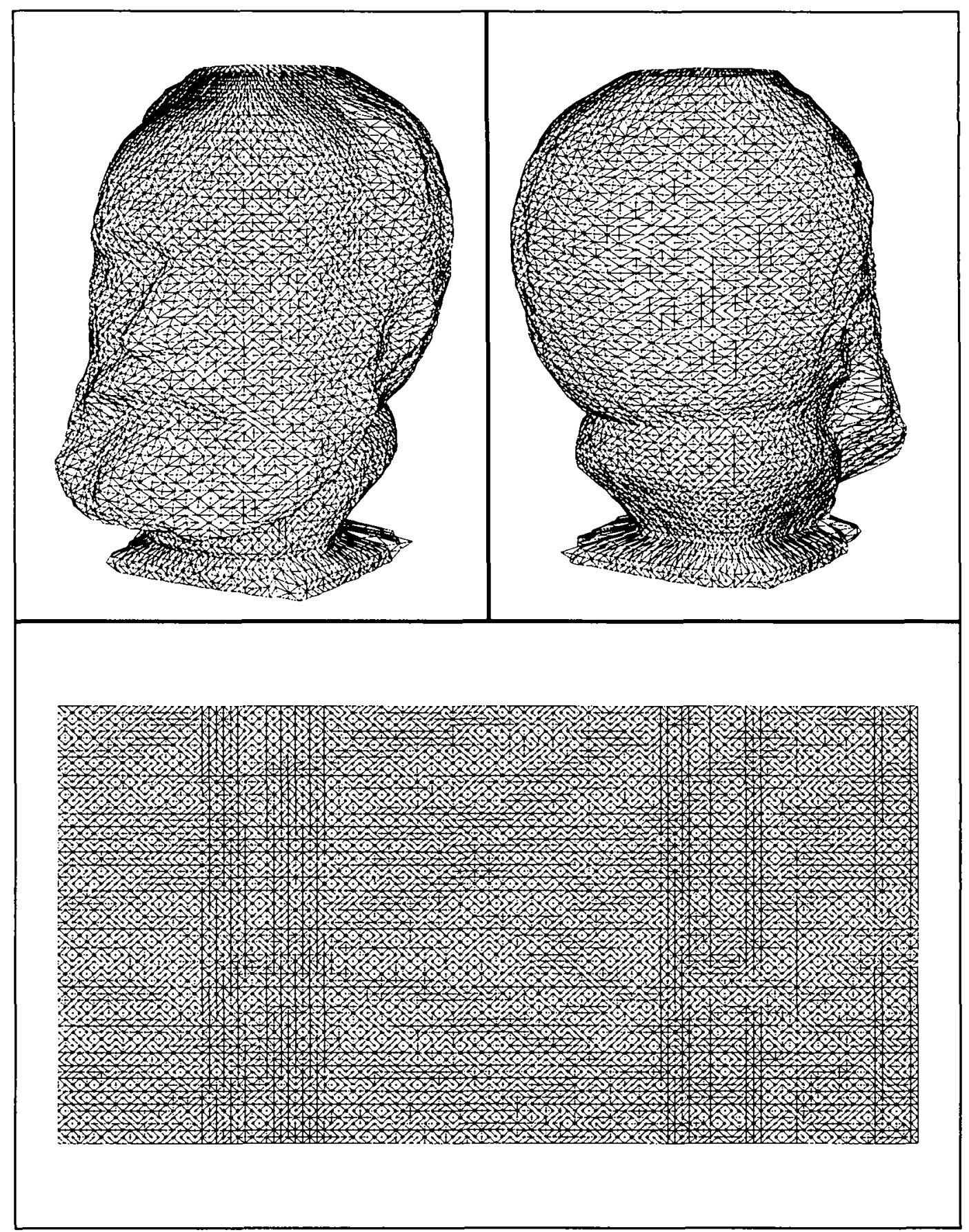

FIG. 10 - Maillage régulier (Hugo).

RR $n^{\circ} 2944$ 


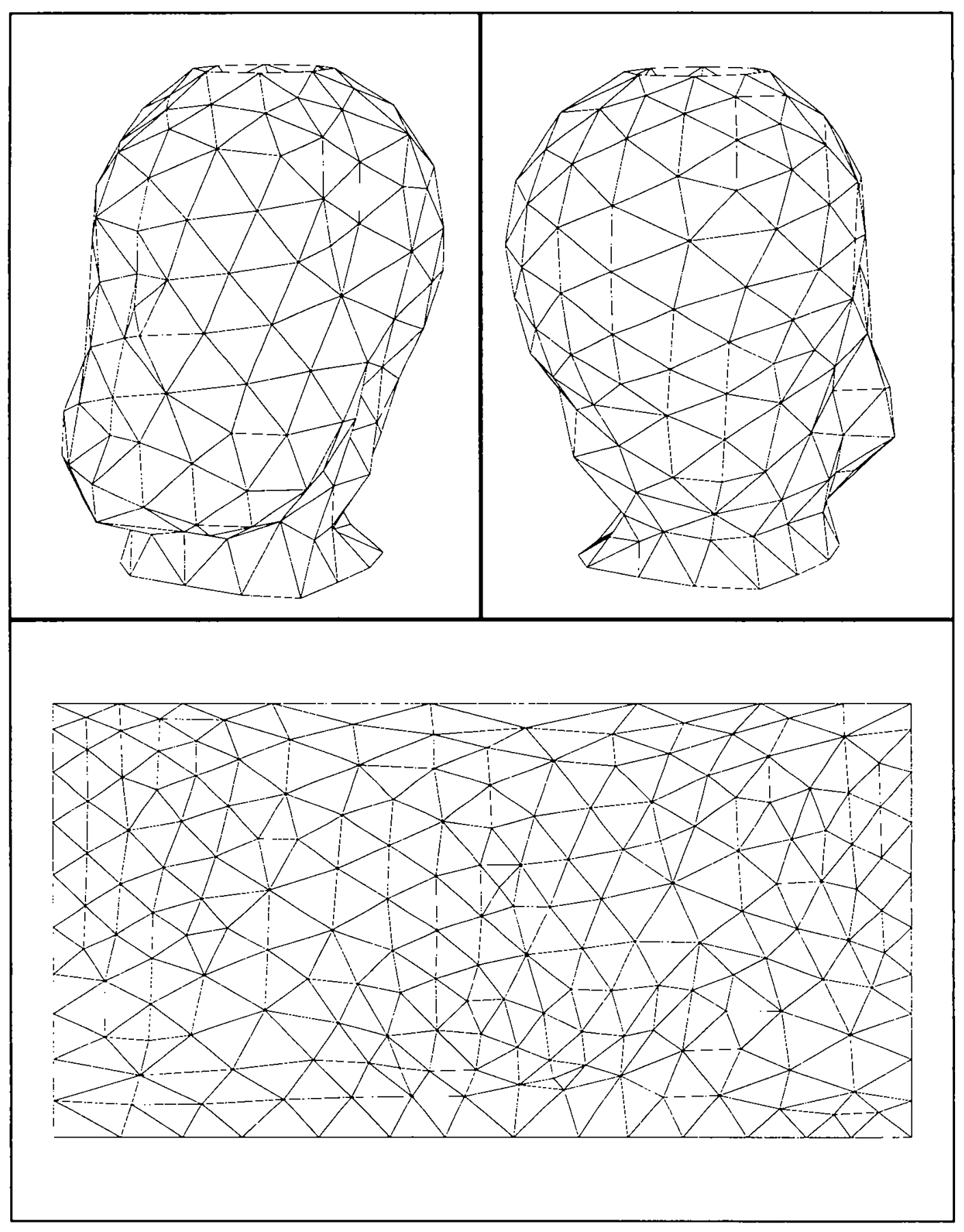

FIG. 11 - Maillage uniforme $h$ (Hugo). 


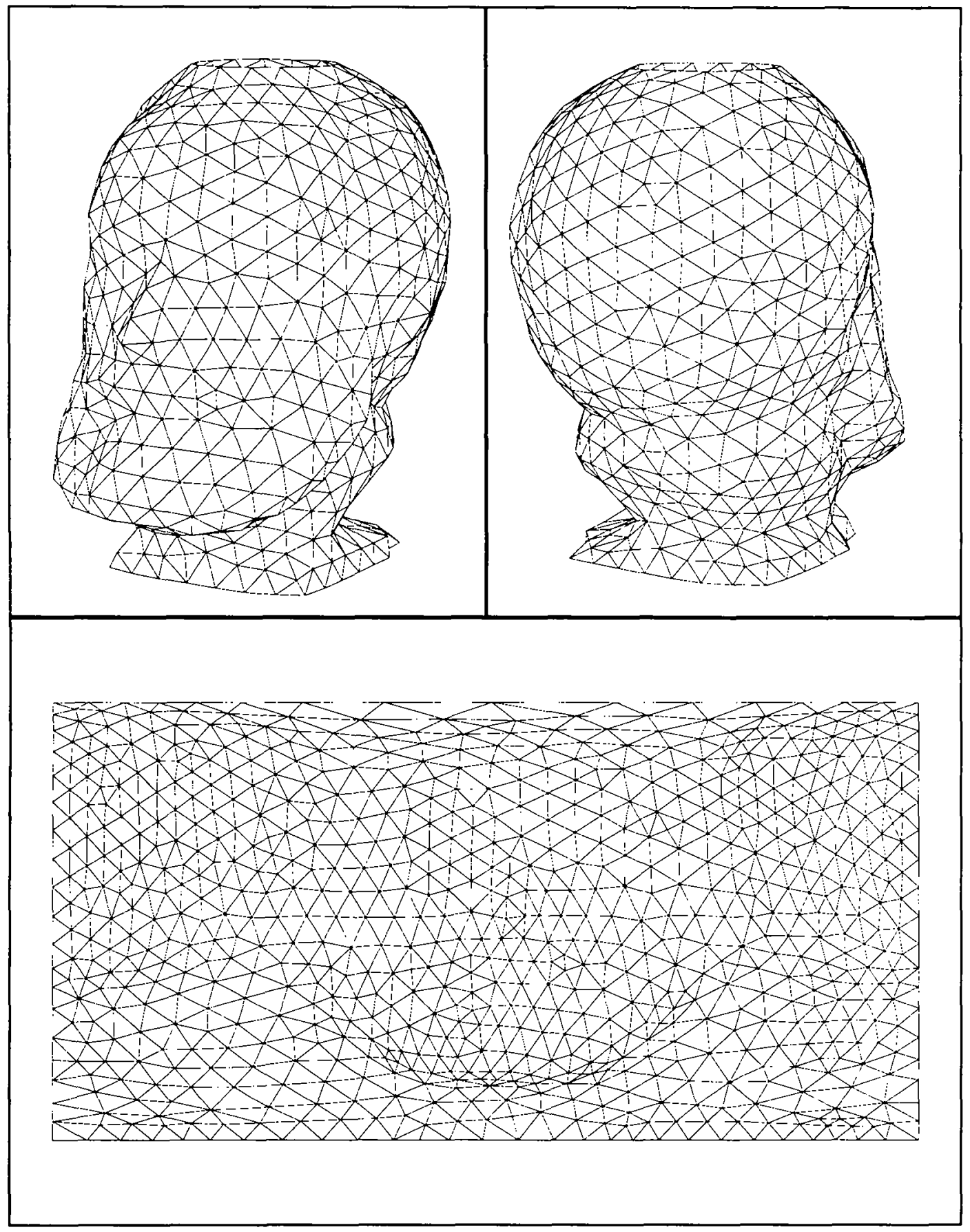

FIG. 12 - Maillage uniforme $\frac{h}{2}$ (Hugo).

RR $\mathrm{n}^{\circ} 2944$ 


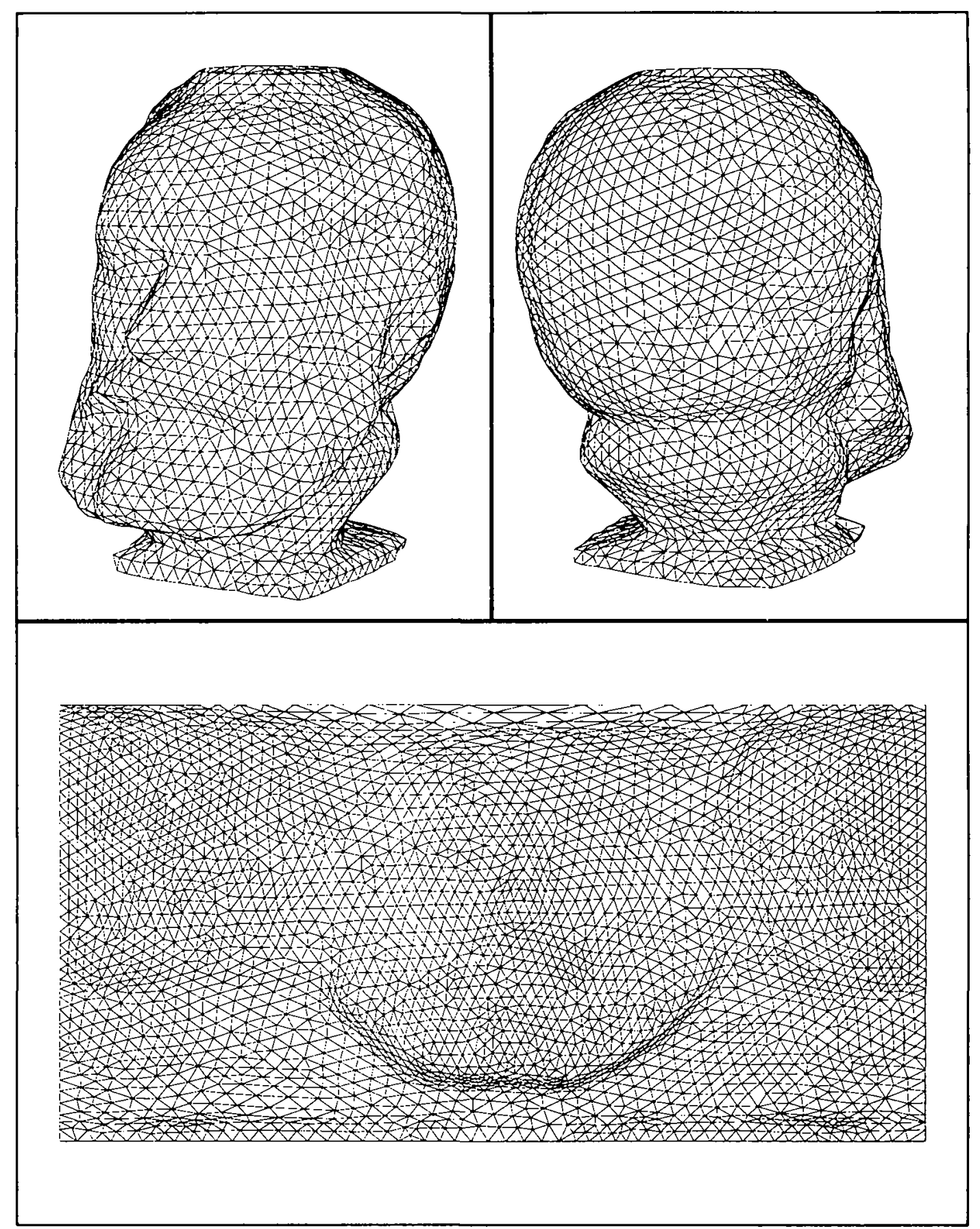

FIG. 13 - Maillage uniforme $\frac{h}{4}$ (Hugo). 


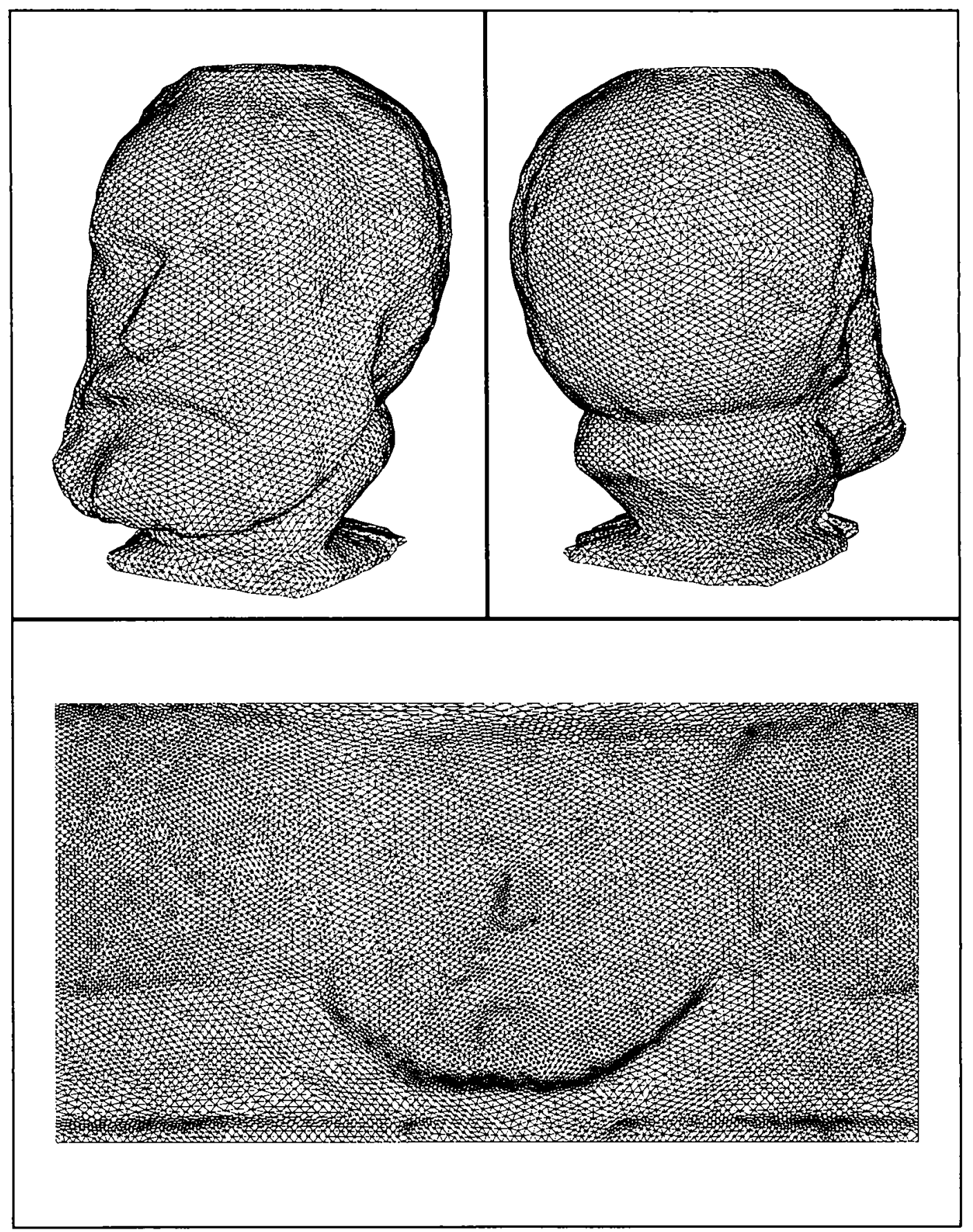

FIG. 14 - Maillage uniforme $\frac{h}{8}$ (Hugo).

$\mathrm{RR} \mathrm{n}^{\circ} 2944$ 


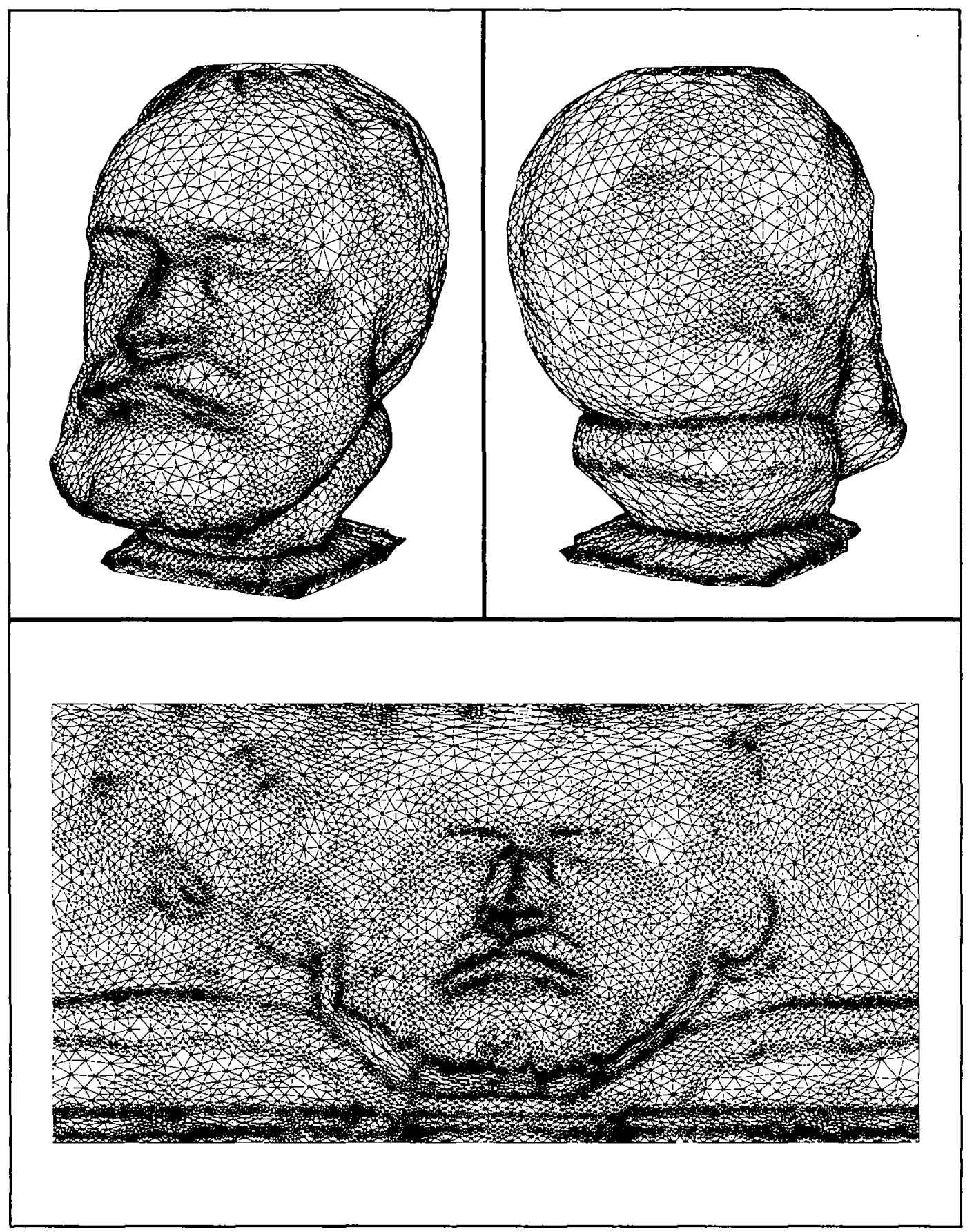

FIG. 15 - Maillage géométrique isotrope (Hugo). 




FIG. 16 - Maillage géométrique anisotrope (Hugo).

RR $n^{\circ} 2944$ 


\begin{tabular}{|l|c|c|c|c|}
\hline Champ & $n p$ & $n t$ & $t_{C P U}$ & $\mathrm{e}$ \\
\hline uniforme $h$ & 203 & 362 & 0.3 & 127. \\
\hline uniforme $\frac{h}{2}$ & 755 & 1427 & 1. & 135. \\
\hline uniforme $\frac{h}{4}$ & 2895 & 5625 & 2.5 & 149. \\
\hline uniforme $\frac{h}{8}$ & 11505 & 22681 & 8. & 150. \\
\hline géométrique isotrope & 12569 & 24847 & 10. & 147. \\
\hline géométrique anisotrope & 5925 & 11584 & 7. & 1686. \\
\hline
\end{tabular}

TAB. 7 - Statistiques relatives aux maillages du buste de Hugo.

\subsubsection{Maillage du buste de Voltaire}

On utilise la deuxième méthode. La figure 17 montre un maillage régulier de la couronne Voltaire ainsi que son report sur la surface, servant d'espace de contrôle pour les autres maillages gouvernés de l'objet. Les figures 18 et 19 montrent des maillages uniformes avec des pas proportionnels. Les figures 20 et 21 montrent des maillages géométriques. Les statistiques relatives à ces maillages sont reportées dans le tableau 8.

\begin{tabular}{|l|c|c|c|c|}
\hline Champ & $n p$ & $n t$ & $t_{C P U}$ & $\mathrm{e}$ \\
\hline uniforme $h$ & 3684 & 7364 & 2. & 360. \\
\hline uniforme $\frac{h}{2}$ & 14029 & 28052 & 11. & 360. \\
\hline géométrique isotrope & 26141 & 52276 & 25. & 350. \\
\hline géométrique anisotrope & 10190 & 20374 & 11. & 290. \\
\hline
\end{tabular}

TAB. 8 - Statistiques relatives aux maillages du buste de Voltaire. 


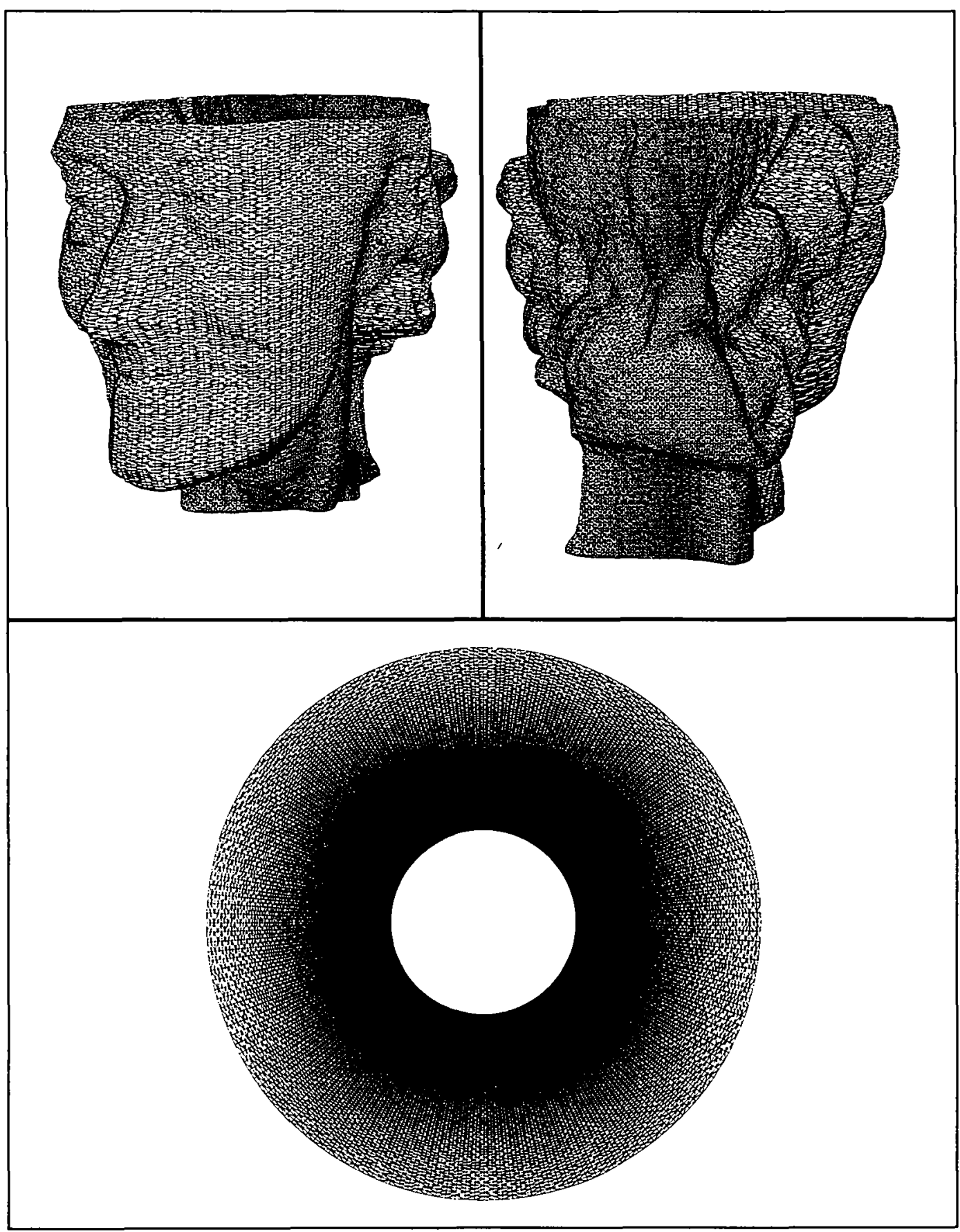

FIG. 17 - Maillage régulier (Voltaire).

RR n ${ }^{\circ} 2944$ 


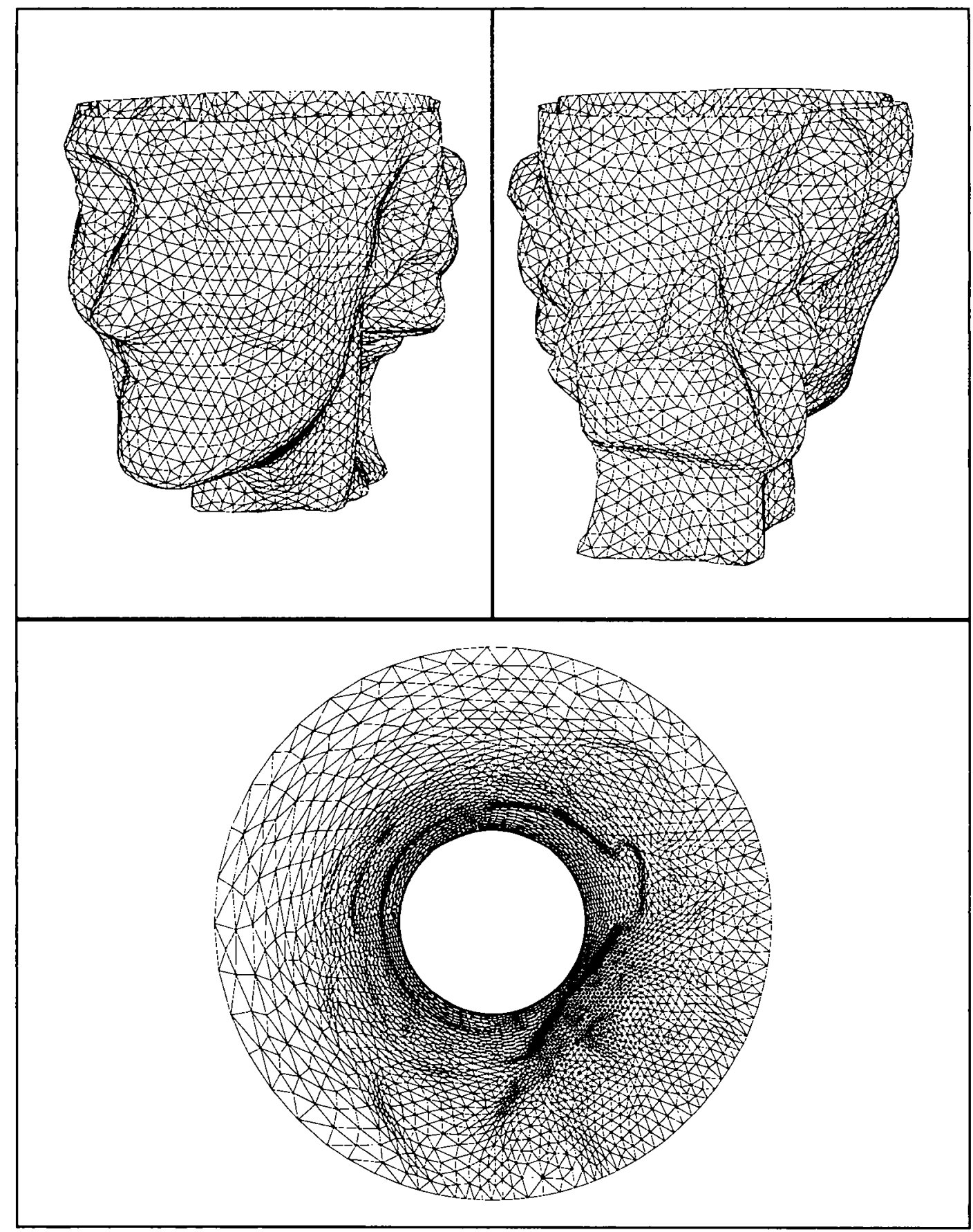

FIG. 18 - Maillage uniforme $h$ (Voltaire). 


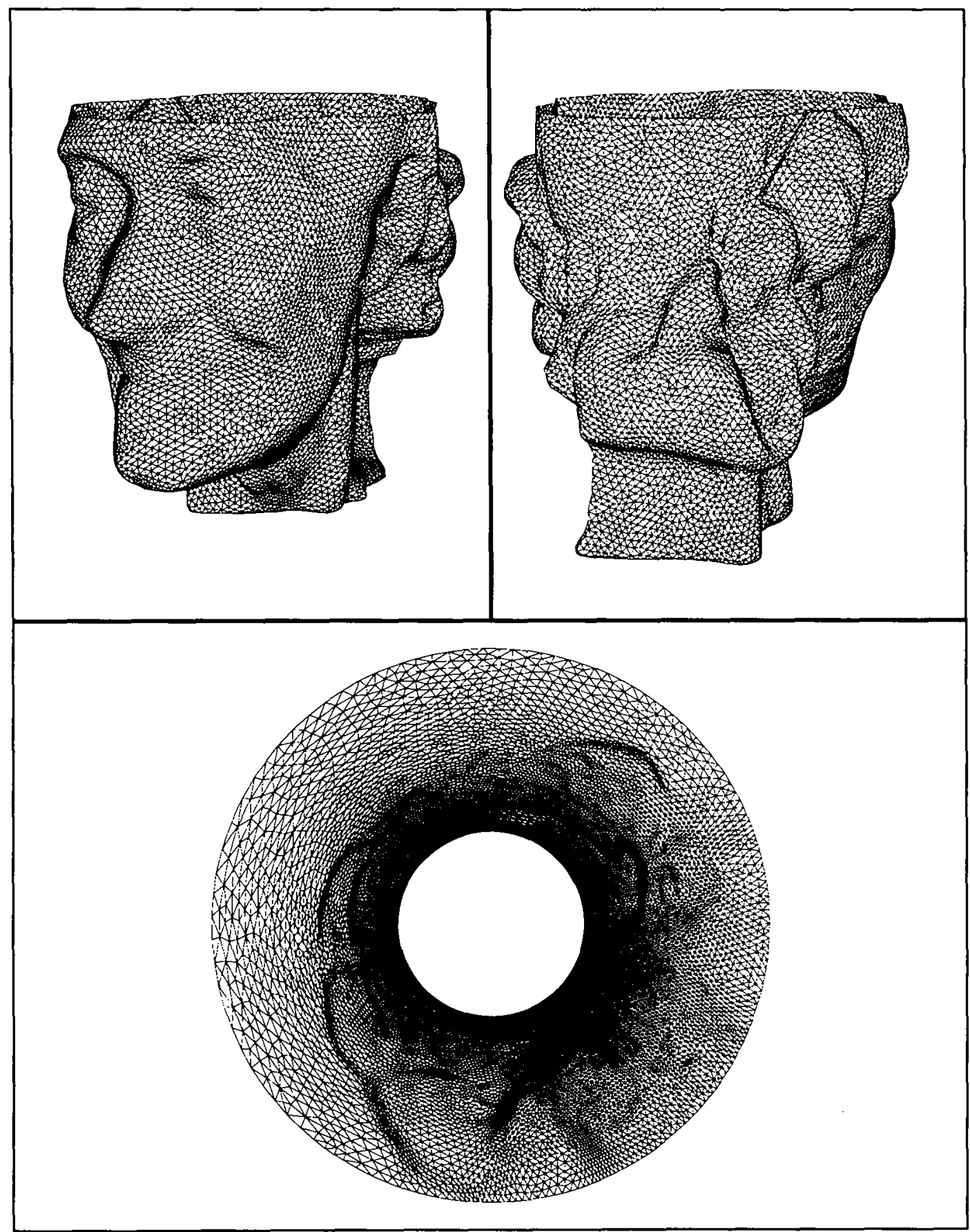

FIG. 19 - Maillage uniforme $\frac{h}{2}$ (Voltaire).

$\mathrm{RR} \mathrm{n}^{\circ} 2944$ 


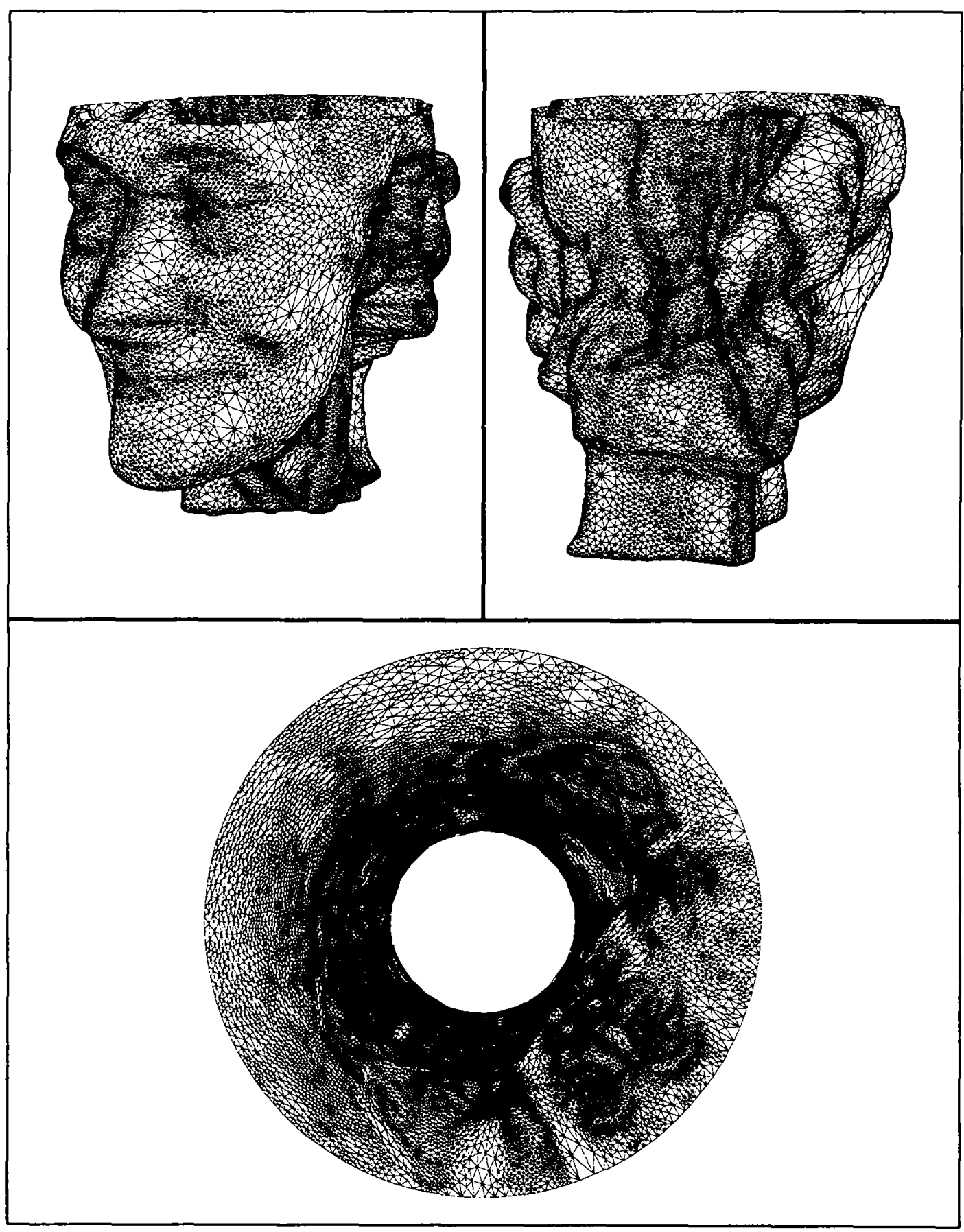

FIG. 20 - Maillage géométrique isotrope (Voltaire). 


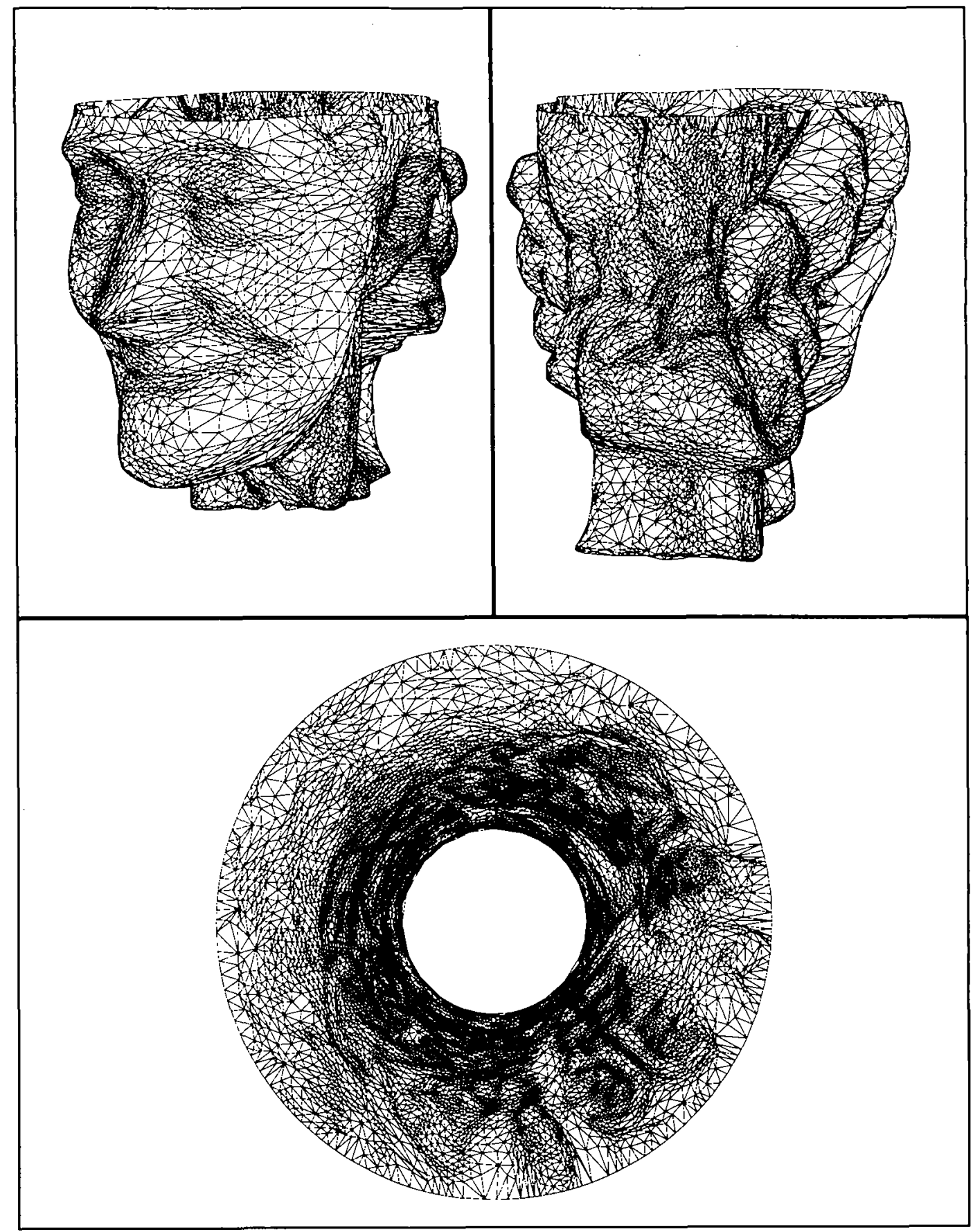

FIG. 21 - Maillage géométrique anisotrope (Voltaire).

$\mathrm{RR} \mathrm{n}^{\circ} 2944$ 


\section{Conclusions}

Plusieurs types de maillages de surfaces ont été présentés pour un exemple de surface paramétrique. En particulier, le maillage gouverné géométrique de la surface. Une application de la méthode au maillage de surfaces cylindriques a été donnée. Le même concept a été appliqué au cas du maillage d'un objet cylindrique numérisé, pour lequel plusieurs exemples ont été donnés. Le problème de maillage triangulaire semble être résolu, sa généralisation au cas des maillages par quadrangles fait l'objet de la partie III de ce rapport. Pour conclure, il reste à envisager le problème du maillage d'une surface paramétrique par morceaux, pour lequel le maillage doit comprendre deux étapes, maillage des interfaces et maillages des cartes. Ce travail est en cours de développement.

\section{Références}

[1] H.Borouchaki ET P.L.George, Maillage de surfaces paramétriques. Partie I: Aspects théoriques, Rapport de recherche INRIA, RR-2928, 1996.

[2] H.Borouchaki, P.L.George, F.Hecht, P.Laug and E.Saltel, Delaunay Mesh Generation Governed by Metric Spccifications. Part I: Algorithms., à paraître dans Finite Elements in Analysis and Design.

[3] F.Schmitt, H.Maître, A.Clainchard and J.Lopez-Krahm, Acquisition and Representation of Real Object Surface Data, SPIE Proceedings Vol. 602 of Biostereometrics'85 Conference, Cannes, France, 2-6 December, 1985. 
Unité de recherche INRIA Rocquencourt

Domaine de Voluceau - Rocquencourt - B.P. 105 - 78153 Le Chesnay Cedex (France)

linite de recherche INRIA Lorraine - Technopôle de Nancy-Brabois - Campus scientifique 615, rue du Jardin Botanique - B.P. 101 - 54602 Villers lès Nancy Cedex (France)

Lnité de recherche INRIA Rennes - IRISA, Campus universitaire de Beaulieu 35042 Rennes Cedex (France)

Lnite de recherche INRIA Rhóne-Alpes 46. avenue Félix Viallet - 3803I Grenoble Cedex I (France)

Unite de recherche INRIA Sophia Antipolis - 2004, route des Lucioles-B.P. 93 - 06902 Sophia Antipolis Cedex (France)

Éditeur

INRIA - Domaine de Voluceau - Rocquencourt - B.P. 105 - 78153 Le Chesnay Cedex (France)

ISSN ()249- 6399 